SANDIA REPORT

Unlimited Release

Printed August 2004

\title{
External Second Gate-Fourier Transform Ion Mobility Spectrometry
}

Edward E. Tarver, Ph.D.

Analytical Materials Sciences Department, Sandia National Laboratories

Livermore, California 94551-0969 USA

Prepared by

Sandia National Laboratories

Albuquerque, New Mexico 87185 and Livermore, California 94550

Sandia is a multiprogram laboratory operated by Sandia Corporation, a Lockheed Martin Company, for the United States Department of Energy's

National Nuclear Security Administration under Contract DE-AC04-94-AL85000.

Approved for public release; further dissemination unlimited.

Sandia National Laboratories 
Issued by Sandia National Laboratories, operated for the United States Department of Energy by Sandia Corporation.

NOTICE: This report was prepared as an account of work sponsored by an agency of the United States Government. Neither the United States Government, nor any agency thereof, nor any of their employees, nor any of their contractors, subcontractors, or their employees, make any warranty, express or implied, or assume any legal liability or responsibility for the accuracy, completeness, or usefulness of any information, apparatus, product, or process disclosed, or represent that its use would not infringe privately owned rights. Reference herein to any specific commercial product, process, or service by trade name, trademark, manufacturer, or otherwise, does not necessarily constitute or imply its endorsement, recommendation, or favoring by the United States Government, any agency thereof, or any of their contractors or subcontractors. The views and opinions expressed herein do not necessarily state or reflect those of the United States Government, any agency thereof, or any of their contractors.

Printed in the United States of America. This report has been reproduced directly from the best available copy.

Available to DOE and DOE contractors from

U.S. Department of Energy

Office of Scientific and Technical Information

P.O. Box 62

Oak Ridge, TN 37831

Telephone: $\quad$ (865) 576-8401

Facsimile: $\quad$ (865) 576-5728

E-Mail: $\quad$ reports@adonis.osti.gov

Online ordering: http://www.doe.gov/bridge

Available to the public from

U.S. Department of Commerce

National Technical Information Service

5285 Port Royal Rd

Springfield, VA 22161

Telephone: $\quad$ (800) 553-6847

Facsimile: (703) 605-6900

E-Mail: $\quad$ orders@ntis.fedworld.gov

Online order: $\quad$ http://www.ntis.gov/help/ordermethods.asp?loc=7-4-0\#online

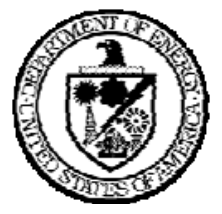


SAND

Unlimited Release

Printed August 2004

\title{
External Second Gate-Fourier Transform Ion Mobility Spectrometry
}

\author{
Edward E. Tarver, Ph.D. \\ Analytical Materials Sciences Department \\ Sandia National Laboratories \\ P.O. Box 969 - MS 9403 \\ Livermore, California 94551-0969 USA
}

\begin{abstract}
Ion mobility spectrometry (IMS) is recognized as one of the most sensitive and versatile techniques for the detection of trace levels of organic vapors. IMS is widely used for detecting contraband narcotics, explosives, toxic industrial compounds and chemical warfare agents. Increasing threat of terrorist attacks, the proliferation of narcotics, Chemical Weapons Convention treaty verification as well as humanitarian de-mining efforts has mandated that equal importance be placed on the analysis time as well as the quality of the analytical data. ${ }^{(1)}$ IMS is unrivaled when both speed of response and sensitivity has to be considered. ${ }^{(2)}$ With conventional (signal averaging) IMS systems the number of available ions contributing to the measured signal to less than $1 \%$. Furthermore, the signal averaging process incorporates scan-to-scan variations decreasing resolution. With external second gate Fourier Transform ion mobility spectrometry (FT-IMS), the entrance gate frequency is variable and can be altered in conjunction with other data acquisition parameters to increase the spectral resolution. The FT-IMS entrance gate operates with a $50 \%$ duty cycle and so affords a 7 to 10 -fold increase in sensitivity. Recent data on high explosives are presented to demonstrate the parametric optimization in sensitivity and resolution of our system.
\end{abstract}


(Page Intentionally Left Blank) 
Abstract

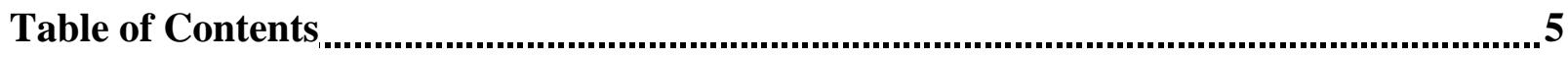

List of Illustrations

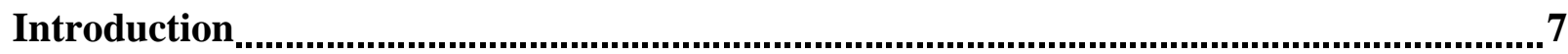

Section I: Fourier Transform Ion Mobility Spectrometry .............................................

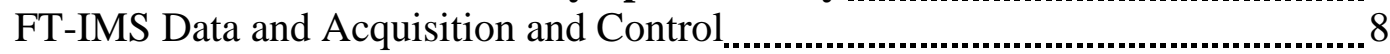

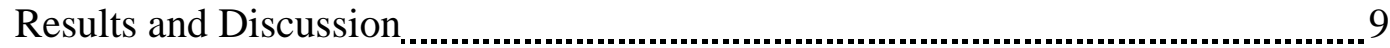

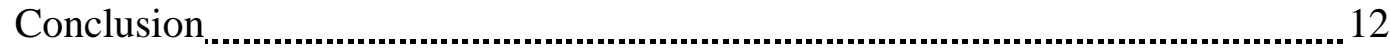

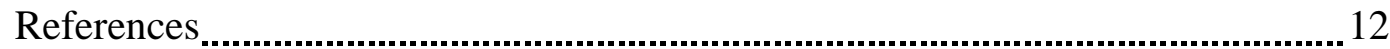

Section II: Data Acquisition and Control System for Fourier Transform Ion Mobility Spectrometer............................................................................................13

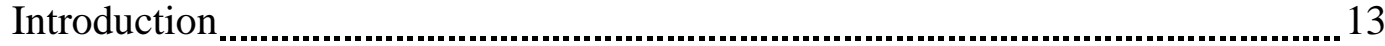

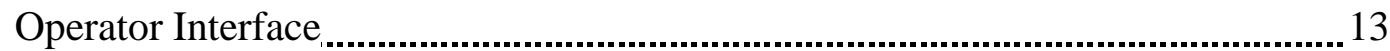

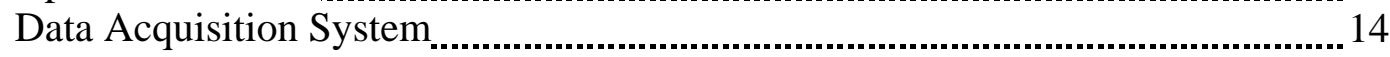

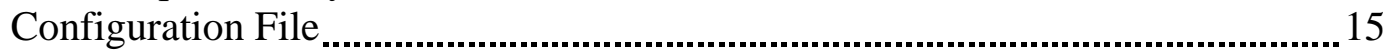

Drift Tube Test Bed

Section III: Size Evaluation of Drift Tubes for Fourier Transform Ion Mobility

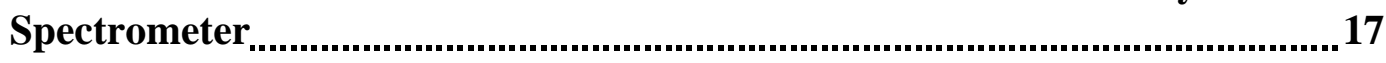

Executive Summary

Findings

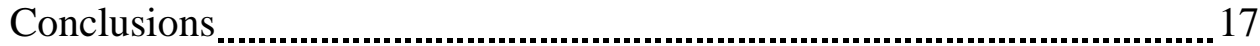

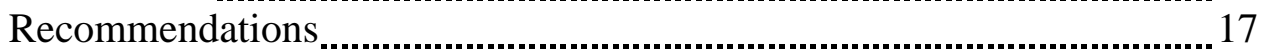

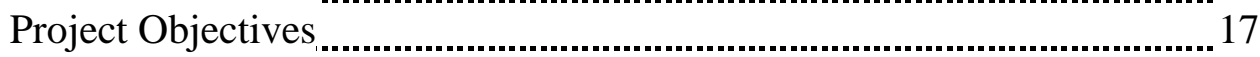

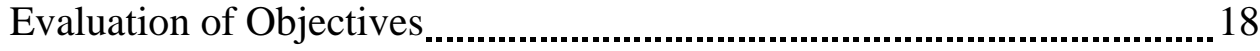

Experimental Set-Up............................................................................. 19

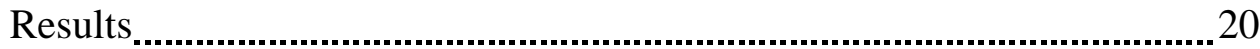

Summary and Conclusions......................................................................... 28 


\section{Section 1}

\section{List of Illustrations}

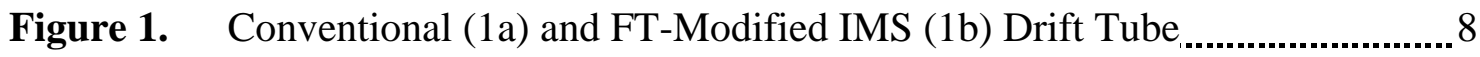

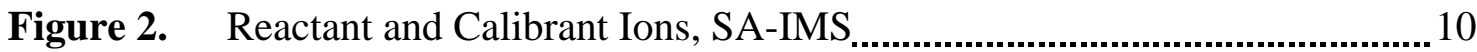

Figure 3. Reactant and Calibrant Ions, FT-IMS (10 kHZ) .....................................

Figure 4. Reactant and Calibrant Ions, FT-IMS (20kHZ and 40kHZ)..................... 10

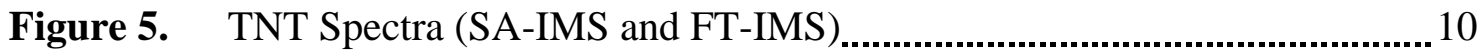

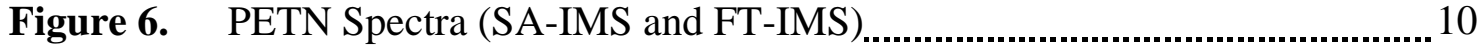

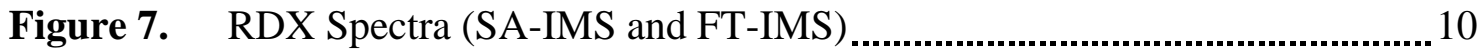

Table 1. Peak Resolution vs. Peak Aspect Ratio................................................. 12

\section{Section II}

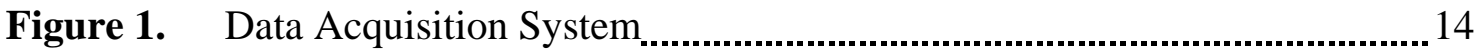

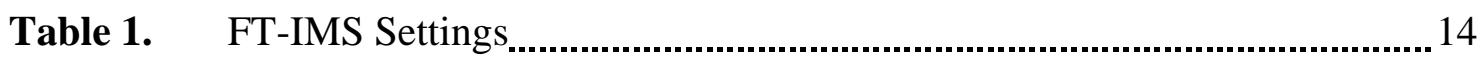

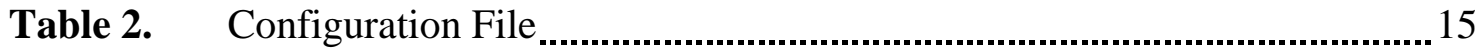

Figure 2. Drift Tube Test Bed $\ldots$

\section{Section III}

Figure 1. Source, Ion Gate and Drift Tube Schematic ........................................ 19

Figure 2. Reactant Ion Drift Time vs. Drift Voltage ............................................... 20

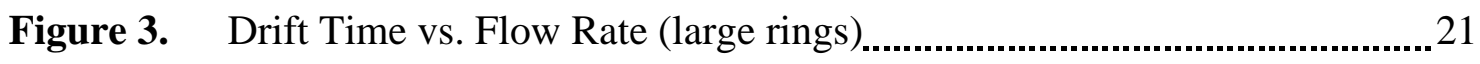

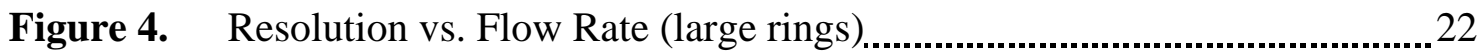

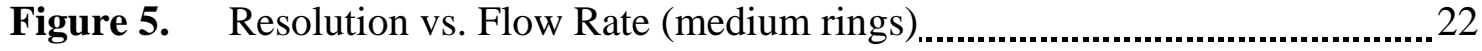

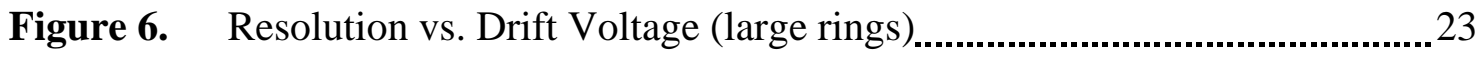

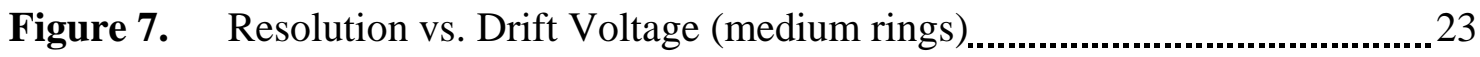

Figure 8. Resolution vs. Drift Voltage (WSU rings).............................................. 24

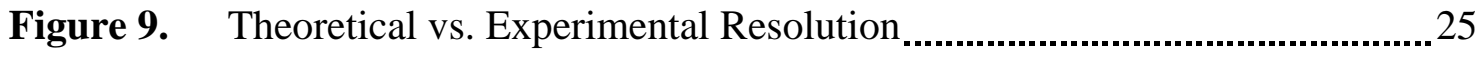

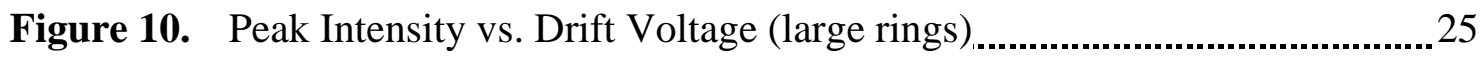

Figure 11. Peak Intensity vs. Drift Voltage (medium rings) ..................................26

Figure 12. Peak Intensity vs. Drift Voltage (WSU rings)

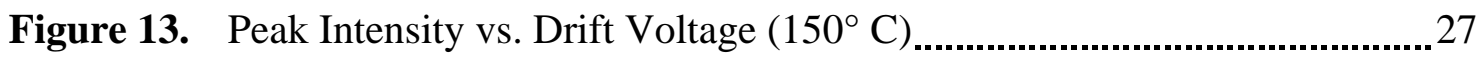

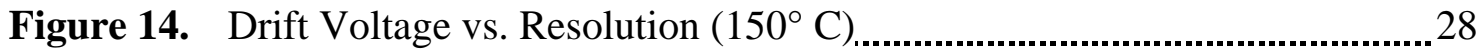




\section{External Second Gate-Fourier Transform Ion Mobility Spectrometry}

\section{Introduction}

Ion mobility spectrometry is based on the atmospheric pressure ionization of sample vapors and the subsequent separation of individual components of the sample mixture as they are accelerated by an electric field gradient and transit a time-of-flight drift tube against a neutral counter-flowing gas stream. The sample vapors are drawn into the detector, and ionized through proton transfer or electron capture reactions, depending on the polarity of electric field, to form "product ions". The ions are periodically pulsed into the separation region of the spectrometer by an electronic gating grid where they establish terminal velocities under the influence of the field gradient and are differentiated according to their characteristic mobility's against the counterflowing drift gas. The separation region begins at the gating grid and terminates at a collecting electrode. The collecting electrode is a Faraday plate. An aperture grid is located just ahead of the collecting electrode to capacitatively de-couple the approaching ion cloud and prevent peak broadening due to premature response. The spectrum of ion arrival times at the collecting electrode indicates the relative mobility of each sample ion through the separation region. Response in IMS is measured as a function of ion current produced versus the ion arrival time at the collecting electrode after each 20-30 ms analytical cycle. In typical IMS instruments, spectra are obtained by pulsing open the electronic gating grid (entrance gate) of the time-of-flight drift tube (separation region) for $0.2 \mathrm{~ms}$ to admit an ion pulse. This brief $0.2 \mathrm{~ms}$ entrance gate pulse represents $\leq 1 \%$ of the total $20-25$ ms analytical "duty cycle" of the instrument. With conventional IMS, more than $99 \%$ of the ions formed are discarded and never reach the detector as a result of the $1 \%$ duty cycle of the entrance gate. ${ }^{(3)}$ Therefore, to achieve an acceptable signal-to-noise ratio $(\mathrm{S} / \mathrm{N})$ with such a low duty cycle, multiple scans are taken and summed with a computer. This summation or "signal-averaging" introduces an additional source of peak broadening in conventional, signal-averaging IMS (SA-IMS).

\section{Section I: Fourier Transform Ion Mobility Spectrometry}

An alternative method of signal acquisition based on ion mobility spectrometry utilizes a twogate design and involves the Fourier transformation of a frequency domain ion mobility interferogram to recover the normal time domain ion mobility spectrum. ${ }^{(4)}$ The interferogram is generated as the ions pulsed into the spectrometer by the entrance gate interact with the second simultaneously pulsed "exit gate". The ions reach the second gate after the time-of-flight delay for ion transit through the separation region. An interference signal is produced that rises and falls depending on the degree to which the second gate is opened or is closing as the ions arrive. For an ion to have maximum contribution to the signal it must be traveling at a constant velocity just matching the gate opening frequency of the second gate as it is swept. Minimal signal contribution is made if the ion reaches the gate as it closes. For a mixed sample containing a broad range of ion velocities there must be a broad range of gate opening frequencies to record the signal for each of the ion velocities. This is accomplished by pulsing open the gates with a square wave at continually increasing frequency from a few Hertz up to tens of kilo-Hertz over the analytical cycle. In addition, since the waveform is square, the gates are open for the same 
amount of time they are closed as they increase in frequency over the entire analytical cycle. All of the velocity information about each of the ions sampled is encoded in the interference signal (the interferogram). The mathematical relationship between the ion's velocity and the gate frequency allows for the Fourier transformation of the frequency interferogram and the recovery of the time domain ion mobility spectrum. In the mid-nineteen eighties the earliest FT-IMS designs by Hill, et al. ${ }^{(4)}$, had both of the two electronic gating grids located inside the drift tube. Half of the initial ion population approaching the first gate would be admitted into the drift tube (separation region) and of this population only half would pass through the exit gate and reach the Faraday plate. With this design, a net 25\% duty cycle was achieved and the signal-to-noise improved by a factor of five (Increase in $\mathrm{S} / \mathrm{N}=\sqrt{25}$ ) over conventional IMS systems. In our Sandia "external second gate" design we have eliminated the internal exit gate and developed a FT-IMS system capable of achieving a 50\% duty cycle as well as higher resolution than conventional IMS. ${ }^{(5,6)}$ By modifying a single gate, signal-averaging instrument we have configured a two-gate FT-IMS instrument in which the function of the internal, hardware exit gate is performed externally, outside of the drift tube, in the electronics. In other words, we have developed a "virtual”, external second gate. This allows our Fourier Transform adapted IMS to sample fifty times more ions than the conventional single-gate, signal averaging designs. Without the loss of ion transmission at the second internal hardware exit gate we have observed signal enhancement exceeding the predicted seven fold signal-to-noise enhancement (Increase in $\mathrm{S} / \mathrm{N}=\sqrt{50} \approx 7$ ). We have demonstrated improved, tunable resolution and increased signal-tonoise compared to both the single gate signal-averaging IMS and the Hill, et al internal hardware, two-gate FT-IMS designs. The electronic and software modifications we have developed enable us to adapt all common single-gate signal-averaging IMS instruments to this external second gate FT-IMS method. Figure 1 below, shows a schematic representation of the time-of-flight drift tube (1A) before and after external second gate modification (1B).

\section{FT-IMS Data Acquisition and Control:}

All analyses were performed on a Barringer Ion Scan Model 400 (Barringer Instruments, New Providence, New Jersey) operated in the explosives (negative ion) detection mode. The high voltage was set at $-2000 \mathrm{~V}$. The drift tube temperature was $105{ }^{\circ} \mathrm{C}$, drift flow $350 \mathrm{~mL} / \mathrm{min}$. The instrument was modified to reform both Fourier Transform-IMS and Signal Averaging-IMS.

\section{A. Conventional IMS Drift Tube}

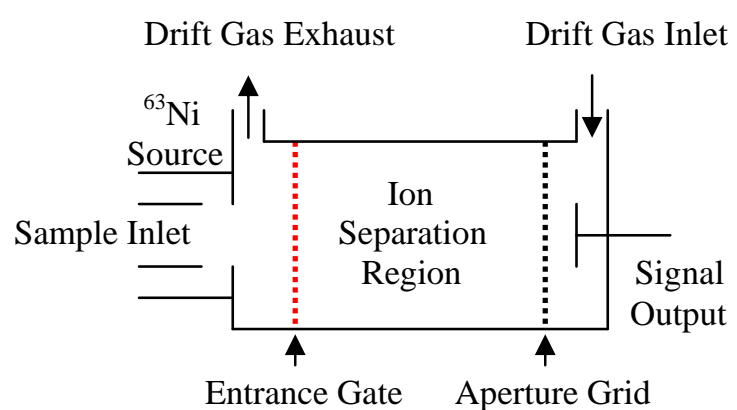

1B. External Second Gate FT-IMS Modification

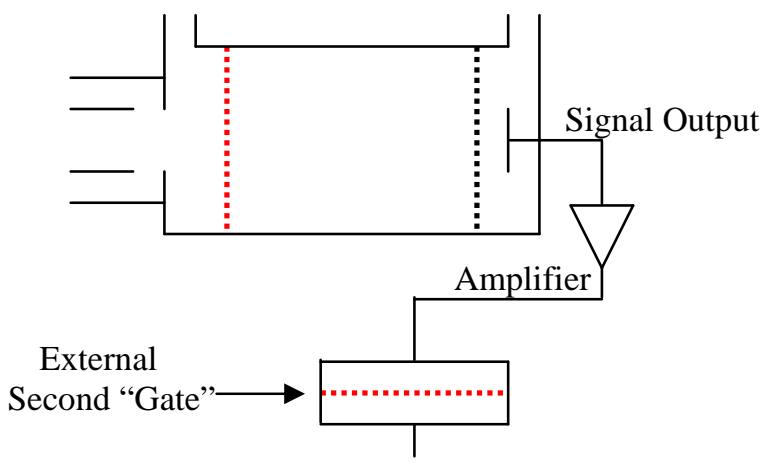

Figure 1. - Conventional (1a) and FT-Modified IMS (1b) Drift Tube 


\section{Results and Discussion:}

Of particular importance is the improved resolving power afforded by the external second gate FT-IMS method. ${ }^{(6)}$ The broad asymmetric peaks observed with IMS are due to clustering and various ion-molecule reactions which alter the velocities of ions during time-of-flight are all summed and recorded in the final signal-averaged IMS spectrum (Figure 2). With the FT-IMS technique only ions of constant velocity contribute to the measured signal so this peak broadening is eliminated. The operator controlled instrumental parameters affecting sensitivity and resolution in external second gate Fourier transform ion mobility spectrometry are: (1) the frequency range selected for the linearly ramped square wave, (2) the number of number of data points/second sampled, (3) the duration selected for the frequency sweep time. Ideally, the speed of the data acquisition system should be maximized to take as much data as fast as possible in order to be able to track short-lived transient species in the detector. This is particularly true with miniaturized IMS drift tubes where transit times are very brief. All spectra displayed and labeled as FT-IMS refer to the external second gate Fourier transform method. Figure 3 compares the spectra of the reactant ion produced by ionization of the drift gas (left) and the calibrant ion used for peak reference (right) using the external second gate FT-IMS method at $10 \mathrm{kHz}$ with that of the SA-IMS technique. Figure 4 displays same two peaks in the FT-IMS mode at $20 \mathrm{kHz}$ and 40 $\mathrm{kHz}$, respectively. In each case the number of data points is 65,536 at 65,536 points/second, resulting in one second scan times. The increase in resolving power with frequency range swept can be traced by monitoring the small shoulder on the left of the predominant reactant ion peak in Figure 4. As the frequency is increased from $20 \mathrm{kHz}$, to $40 \mathrm{kHz}$ the shoulder is clearly separated from the major peak. The higher frequency FT-IMS scans monitor increasingly smaller temporal slices of the ion pulse resulting in decreasing signal intensity. Figure 5 displays the spectra generated from a $100 \mathrm{ppb}$ solution of TNT in acetone deposited onto a sample cartridge and inserted into the IMS after the acetone was evaporated. The signal-averaged IMS spectrum in Figure 5 (red trace) is shown on the same scale as the $10 \mathrm{kHz}$ FT-IMS spectrum (black trace). The total data acquisition time for the signal-averaged IMS spectra was 6.0 seconds. The FTIMS spectrum shown was generated in three seconds total acquisition time. While averaging is not necessary with the FT-IMS method, three one-second scans were taken for this sample. The 10.3 and 12.2 ms peaks will be referenced for method comparison in discussion section. Figure 6 compares the spectra resulting from identical samples of the explosive PETN as a function of parametric settings. Comparing the SA-IMS (6.0 second acquisition) and the FT-IMS (3.0 second acquisition) spectra again demonstrates the striking increase in signal-to-noise achieved using the FT-IMS method.

Figure 7 compares the spectra generated by the two methods from a sample of RDX. The red trace shows the response resulting from 2.0 seconds total acquisition in the conventional signalaveraging mode. The RDX was monitored at the maximum sampling rate using 1.0 seconds scans over a $10 \mathrm{kHz}$ frequency range. The total acquisition time was 3.0 seconds. 


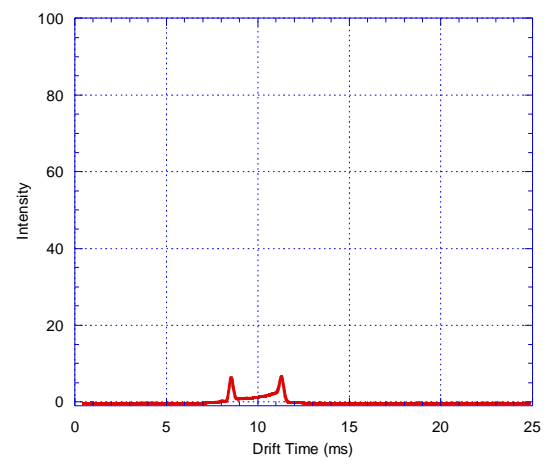

Figure 2. Reactant and Calibrant Ions, SA-IMS

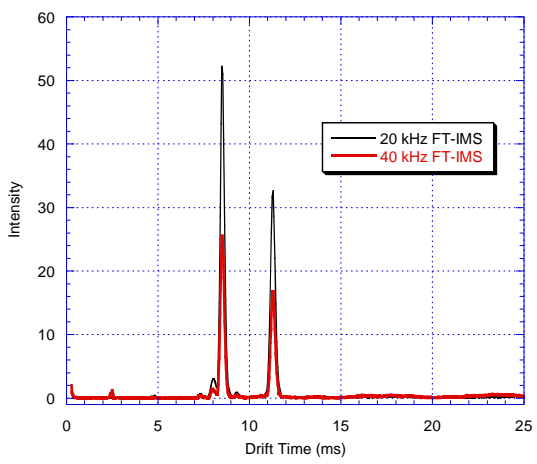

Figure 4. Reactant and Calibrant Ions, FT-IMS (20 kHz and $40 \mathrm{kHz}$ )

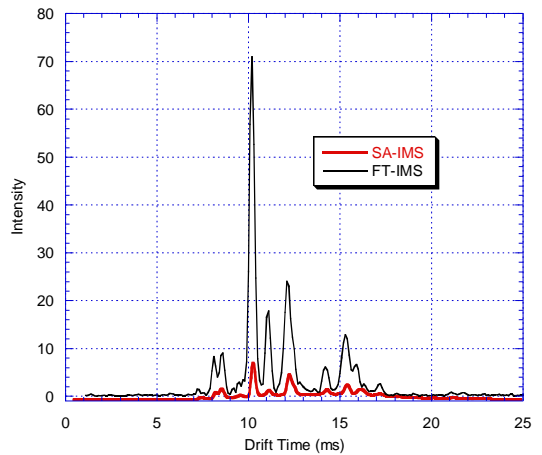

Figure 6. PETN Spectra (SA-IMS and FT-IMS)

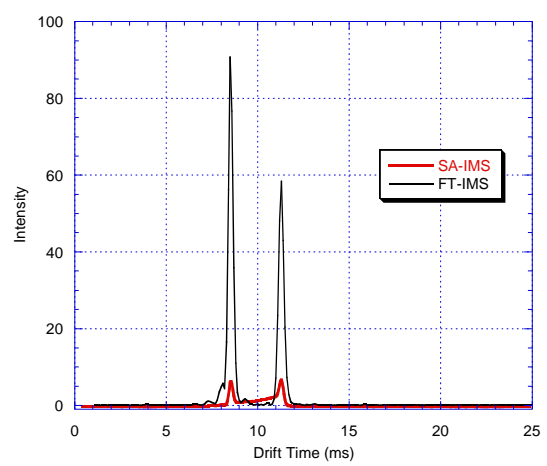

Figure 3. Reactant and calibrant Ions, FT-IMS (10 kHZ)

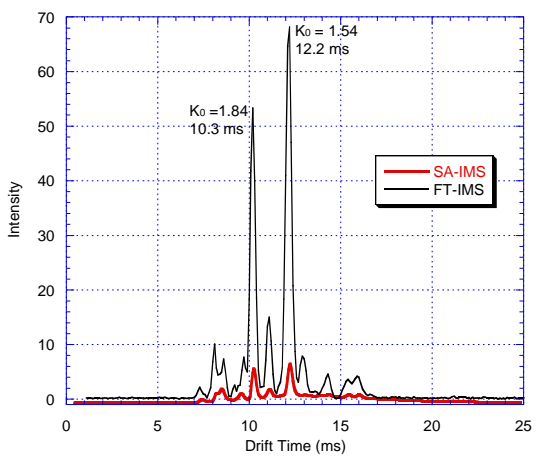

Figure 5. TNT Spectra (SA-IMS and FT-IMS)

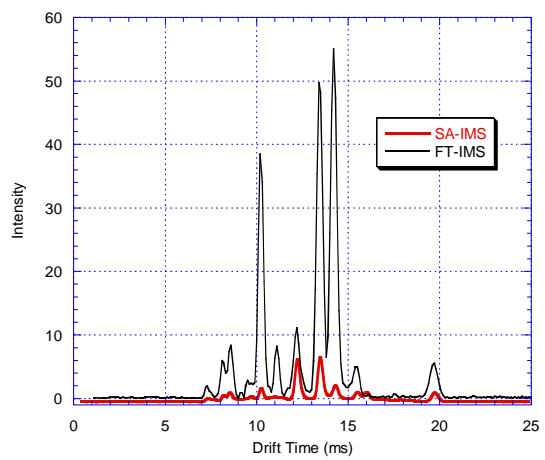

Figure 7. RDX Spectra (SA-IMS and FT-IMS) 
Peak broadening in IMS is primarily determined by the temporal width of the entrance gate pulse (typically about $0.2 \mathrm{~ms}$ ), electric field in-homogeneity, Coulombic repulsion between ions, and diffusional broadening as the ions transit the drift space. ${ }^{(7)}$ Resolution, $\mathrm{R}$, in IMS is traditionally calculated as defined by:

Eq. (1) $\quad \mathrm{R} \equiv \mathrm{t}_{\mathrm{d}} / \mathrm{W}$

where $t_{d}$ is the drift time and $w$ is the full width at half maximum (in drift time) for the peak. This calculation is drift time dependent and also ignores peak asymmetry below half-maximum. As a result, broad peaks with long drift times or peaks that exhibit tailing below half maximum are ascribed misleading resolution values. A more uniform way of describing peak quality is the peak aspect ratio, AR, or the ratio of the peak height to the width at the base where the aspect ratio is calculated by:

Eq. (2) A.R. $=\mathrm{h} / \mathrm{w}_{\mathrm{b}}$

Where, $\mathrm{h}$ is the height at the peak maximum and $\mathrm{w}_{\mathrm{b}}$ is the peak width at the base. The higher the aspect ratio, the easier it is to separate neighboring peaks. FT-IMS can routinely produce peaks with aspect ratios up to 20 times larger than SA-IMS by selecting high sensitivity parametric settings. The combined features of higher sensitivity, resolution, peak aspect ratio, and ease of adaptability to conventional IMS drift tubes and ionization sources has made FT-IMS the solution to the traditional limitations of SA-IMS. Table 1, below compares the resolution, $\mathrm{R}$ and the peak aspect ratio, A.R. for two peaks common to all samples and used for comparison are listed by their respective reduced mobility constants, $\mathrm{K}_{\mathrm{o}}$. which compensate for differences in instrumental design, temperature and pressure. The peaks compared in Table 1 have drift times of 10.3 and $12.2 \mathrm{~ms}$ and are listed by their reduced mobility constants 1.84 and 1.54 respectively, as calculated by:

Eq. (3) $\mathrm{K}_{0}=\mathrm{L}^{2} / \mathrm{t}_{\mathrm{d}} \mathrm{V} \times 273.2 / \mathrm{T} \times \mathrm{P} / 760$

where, $\mathrm{V}$ is the voltage drop across the drift tube, $\mathrm{L}$ is the drift length in $\mathrm{cm}, \mathrm{t}_{\mathrm{d}}$ is the drift time in sec, $\mathrm{P}$ is the drift gas pressure in Torr, and $\mathrm{T}$ is the Temperature. 
Table 1. - Peak Resolution vs. Peak Aspect Ratio

\begin{tabular}{|c|c|c|c|c|c|c|c|c|c|}
\hline & & Peak Re & $=\mathbf{t}_{\mathrm{d}} / \mathbf{w}_{1 / 2}$ & & & Peak As & Ratio ( & $\mathbf{R}=\mathbf{h} / \mathbf{h}$ & \\
\hline Peak $K_{0}$ & $=1.84,1$ & & & & & & & & \\
\hline & & SA-IMS & FT-IM & (Hertz) & & SA-IMS & FT-IM & (Hertz) & \\
\hline Sample: & & & $(10 \mathrm{~K})$ & $(20 K)$ & & & $(10 \mathrm{~K})$ & $(20 \mathrm{~K})$ & \\
\hline & TNT & 40.97 & 30.27 & 36.59 & & 10.74 & 156.8 & 101.6 & \\
\hline & PETN & 41.23 & 28.74 & 39.56 & & 13.68 & 209.8 & 18.88 & \\
\hline & HMX & 41.35 & 28.57 & 40.98 & & 3.02 & 185.6 & 36.56 & \\
\hline & RDX & ------ & 28.84 & 50.92 & & ------ & 113.4 & 31.89 & \\
\hline & HNS & 41.94 & 28.74 & 34.31 & & 5.98 & 188.4 & 130.2 & \\
\hline Averages & & 41.37 & 29.03 & 37.32 & & 8.35 & 170.8 & 63.82 & \\
\hline Peak $K_{0}$ & $=1.54,1$ & & & & & & & & \\
\hline & & SA-IMS & & MS (He & & SA-IMS & & MS (Heı & \\
\hline Sample: & & & $(10 \mathrm{~K})$ & $(20 K)$ & $(40 \mathrm{~K})$ & & $(10 \mathrm{~K})$ & $(20 \mathrm{~K})$ & $(40 \mathrm{~K})$ \\
\hline & TNT & 45.59 & 30.41 & 30.75 & 42.47 & 9.12 & 156.8 & 134.0 & 56.87 \\
\hline & PETN & 38.20 & 37.42 & 41.40 & ----- & 5.68 & 47.14 & 75.90 & ----- \\
\hline & HMX & 42.04 & 31.76 & 41.49 & 65.99 & 7.52 & 147.4 & 56.84 & 29.81 \\
\hline & RDX & 46.33 & ------ & 34.11 & 75.27 & 9.32 & ----- & 17.86 & ------ \\
\hline & $\underline{\mathrm{HNS}}$ & 45.70 & 26.86 & 40.67 & ------ & $\underline{12.8}$ & 51.70 & 77.13 & ------ \\
\hline Averages & & 43.57 & 31.61 & 37.68 & 61.24 & 8.88 & 100.8 & 72.34 & 43.34 \\
\hline
\end{tabular}

\section{Conclusion:}

Fourier transform ion mobility spectrometry (FT-IMS) has several advantages over conventional ion mobility spectrometers. First, the effective percentage of the analytical cycle time wherein sample ions are admitted into the spectrometer is fifty times greater. Second, the phasing action of the two-gate Fourier transform method eliminates the peak tailing due to variations in ion velocities. Third, information about all ion velocities is obtained simultaneously, eliminating the need to average over many analytical cycles. Finally, FT-IMS is adaptable to conventional IMS.

\section{References:}

1. Erickson, B., Anal Chem (1998) Vol. 70 No. 11, 397-400A

2. Corriveau, J. In Analytical methods for Environmental Sampling of Chemical Warfare Agents and their Degradation Products. Watson, A.P.,; Kistner, S., Eds. (1994) 147-488.

3. U.S. Congress, Office of Technology Assessment. Technology Against Terrorism: The Federal Effort (1991) 84-86.

4. Knorr, F.J.; Eatherton, R.L.; Siems, W.F.; Hill, H.H., Anal Chem (1985) Vol 57, 402406.

5. Tarver, E.E.; Stamps, J.; Jennings, R.; Siems, W.F., Int. Journal for Ion Mobility Spectr., (2001) Vol 4, No. 1.

6. Tarver, E.E., Stamps, J.; Jennings, R., Proceedings: $7^{\text {th }}$ International Symposium on the Analysis and Detection of Explosives. Edinburgh, Scotland. June 25, 2001.

7. Siems, W.F.; Ching Wu; Hill, H.H.; Tarver, E.E., Anal Chem (1994) Vol 66, 4195-4201.. Ching Wu, Siems, W.F.; Asbury, G.R.; Hill, H.H., Anal Chem (1998) Vol. 70 No. 23, 4229-2938. 


\section{Section II: Data Acquisition and Control System for Fourier Transform Ion Mobility Spectrometer}

\section{Introduction:}

This is a brief Operator Manual for the prototype External Second Gate-Fourier Transform Ion Mobility Spectrometer, otherwise known as the FT-IMS. The basic controls are explained and the key operational characteristics are documented. Electronically, the FT-IMS works by generating a sweep frequency to modulate the ion mobility spectrometer (IMS) drift tube circuitry. The IMS detector output is modulated by the sweep frequency producing an interferogram that is read with a 12-bit analog to digital converter. The digital signal is Fourier transformed and a peak detection algorithm is applied. Detected peaks are compared with a lookup table of target ions and the relative intensity of any detected target ion is tagged and plotted.

\section{Operator Interface:}

There are seven buttons on the Ft-IMS Data Acquisition interface module. Each button cycles through a specific instrument setting. Once you have set up the instrument acquisition parameters, press the "scan?" button on the far right to button to perform a scan. The progress bar changes to reflect the scan's progress and displays a text message with the current settings.

The leftmost button, the "Function" button has four different values: FFT, Interferogram, Bar Chart, and Save Data. The first three affect the instrument display mode and the fourth is used to save acquired data. When the instrument is in Save mode the rightmost button changes from Scan to Save. Pressing "Save" will save the FFT waveforms and reduced mobility data to drive D:\ as ASCII text files. The file names are generated based on year, month, date, hour, minute, second. 2004_02_28_09_11_45.txt would contain the FFT waveforms and 2004_02_28_09_11_45.txt.mob the mobility data saved on February, 28, 2004 at 9:11:45.

The five buttons in the middle are used to change the instrument settings. In general, increasing the number of samples increases the signal amplitude and increasing the frequency tends to sharpen the peaks. Changing the "\# Scans" sets the number of scans or how many interferograms the instrument will use for measurements. The "Scan Length" set how long each scan will take. To start scanning, push the rightmost "Scan?” button. The instrument will take a series of interferograms and process the data. You can view the acquired interferograms as they are acquired by pressing the "Function" button until the "Interferogram" function is enabled. The Fourier transformed waveform, i.e. the ion mobility spectrum, is viewed with the "FFT" function enabled. After the interferogram has been acquired and Fourier transformed, the peaks on the resulting ion mobility spectrum are interrogated with the peak detection algorithm. The detected peaks are compared with a look up table of the targeted ion-peak identifiers. The relative intensity of any detected target ion is tagged and plotted. This plot is displayed with the "Bar Chart” function. 
Figure 1. - Data Acquisition System - External Second Gate-Fourier Transform Ion Mobility Spectrometer

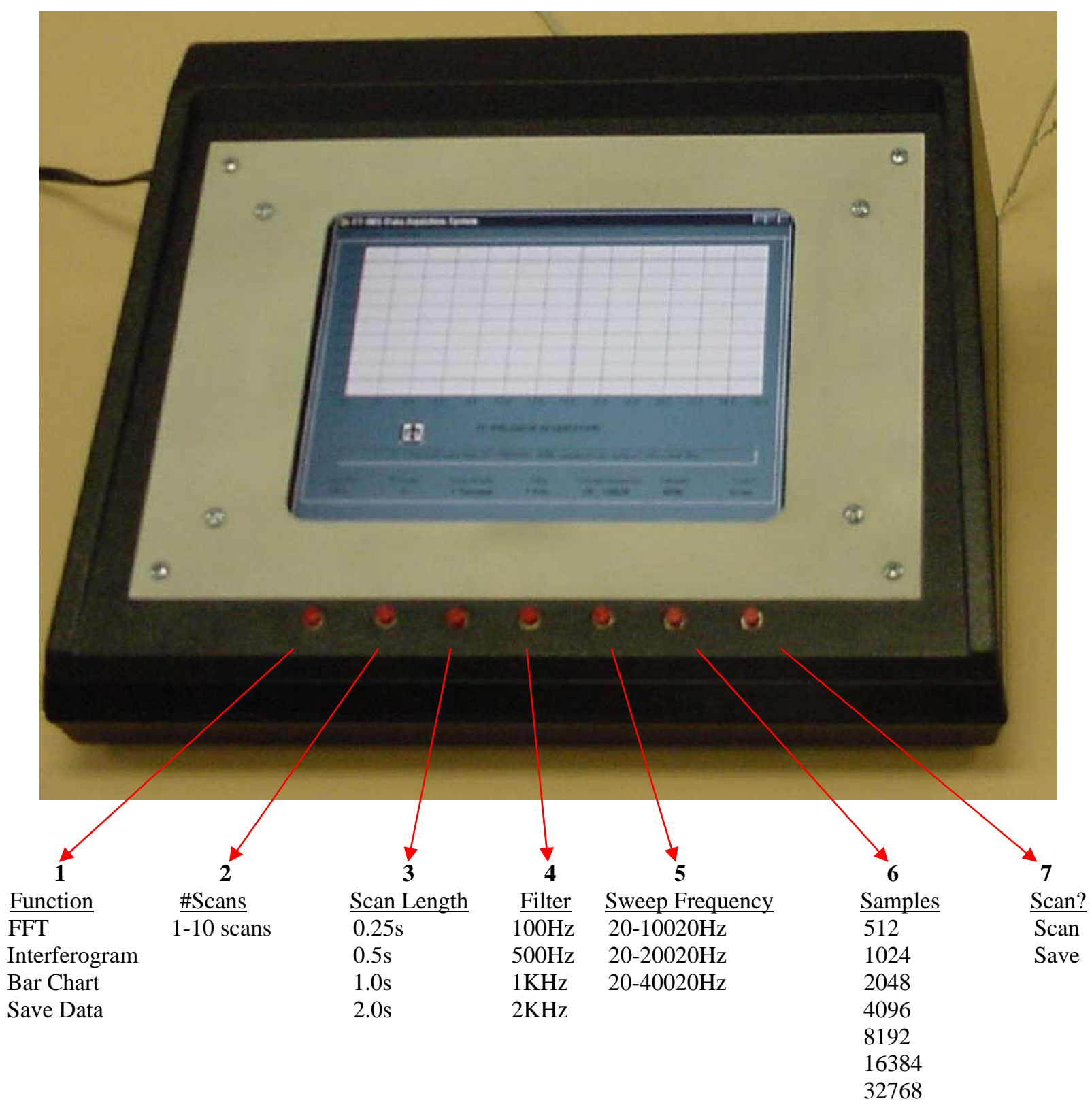

Table 1. - FT-IMS Settings:

\begin{tabular}{|c|c|c|c|}
\hline Scan Length & Filter & Sweep Frequency & $\underline{\text { Samples }}$ \\
\hline $2.0 \mathrm{~s}$ & $\overline{100 \mathrm{~Hz}}$ & $20-10020 \mathrm{~Hz}$ & 1024 \\
\hline $1.0 \mathrm{~s}$ & $1 \mathrm{KHZ}$ & $20-10020 \mathrm{~Hz}$ & 1024 \\
\hline $0.5 \mathrm{~s}$ & $1 \mathrm{KHz}$ & $20-10020 \mathrm{~Hz}$ & 1024 \\
\hline $0.25 \mathrm{~s}$ & $1 \mathrm{KHz}$ & $20-10020 \mathrm{~Hz}$ & 1024 \\
\hline $1.0 \mathrm{~s}$ & $1 \mathrm{KHz}$ & $20-20020 \mathrm{~Hz}$ & 2048 \\
\hline $0.25 \mathrm{~s}$ & $1 \mathrm{KHz}$ & $20-20020 \mathrm{~Hz}$ & 2048 \\
\hline $1.0 \mathrm{~s}$ & $1 \mathrm{KHz}$ & $20-40020 \mathrm{~Hz}$ & 4096 \\
\hline
\end{tabular}




\section{Configuration File:}

When the program starts up it reads a configuration file, Ko.txt. This file is a tab delimited list of target ions, each ions' reduced mobility constant, and upper and lower bounds for detection.

Currently the detection boundary is set to be plus or minus one percent of the input value of Ko. You can add or delete as many ions as you want to this file. Care must be taken not to overlap the ranges of the Ko values or misidentification may result (see Table 2, below).

Table 2. - Configuration File

\begin{tabular}{|l|l|l|l|}
\hline Compound & Ko & Ko-Lower & Ko-Upper \\
\hline DNT & 1.5670 & 1.5513 & 1.5827 \\
\hline TNT & 1.4510 & 1.4365 & 1.4655 \\
\hline RDX-C & 1.3905 & 1.3766 & 1.4044 \\
\hline RDX-N & 1.3150 & 1.3019 & 1.3282 \\
\hline RDX-F & 1.2075 & 1.1954 & 1.2196 \\
\hline RDX-D & 0.9480 & 0.93852 & 0.95748 \\
\hline NO3 & 1.9340 & 1.9147 & 1.9533 \\
\hline PETN-C & 1.1500 & 1.1385 & 1.1615 \\
\hline PETN-N & 1.1018 & 1.0908 & 1.1128 \\
\hline PETN-F & 1.0334 & 1.0231 & 1.0437 \\
\hline NG-C & 1.3395 & 1.3261 & 1.3529 \\
\hline NG-N & 1.2750 & 1.2622 & 1.2878 \\
\hline NG/TNT & 1.4535 & 1.4390 & 1.4680 \\
\hline RDX-NO3 & 1.3150 & 1.3019 & 1.3282 \\
\hline PETN-NO3 & 1.1018 & 1.0908 & 1.1128 \\
\hline cal2 & 1.6500 & 1.6335 & 1.6665 \\
\hline & & & \\
\hline
\end{tabular}

\section{Drift Tube Test Bed:}

A parametrically controlled drift tube test was constructed to evaluate a variety of drift tube configurations using different voltage, temperature and gas flow settings. The drift tubes varied in length, as determined by the number of drift rings, and in internal diameter of the ring. Combinations of internal diameters used on the same tube were also evaluated to see if a focusing effect could be achieved. High voltage capability ranged from 50-3000 Volts, temperature from ambient to 300 degrees Celsius, and gas flows from 50-5000 milliliters per minute. 
Figure 2. - Drift Tube Test Bed
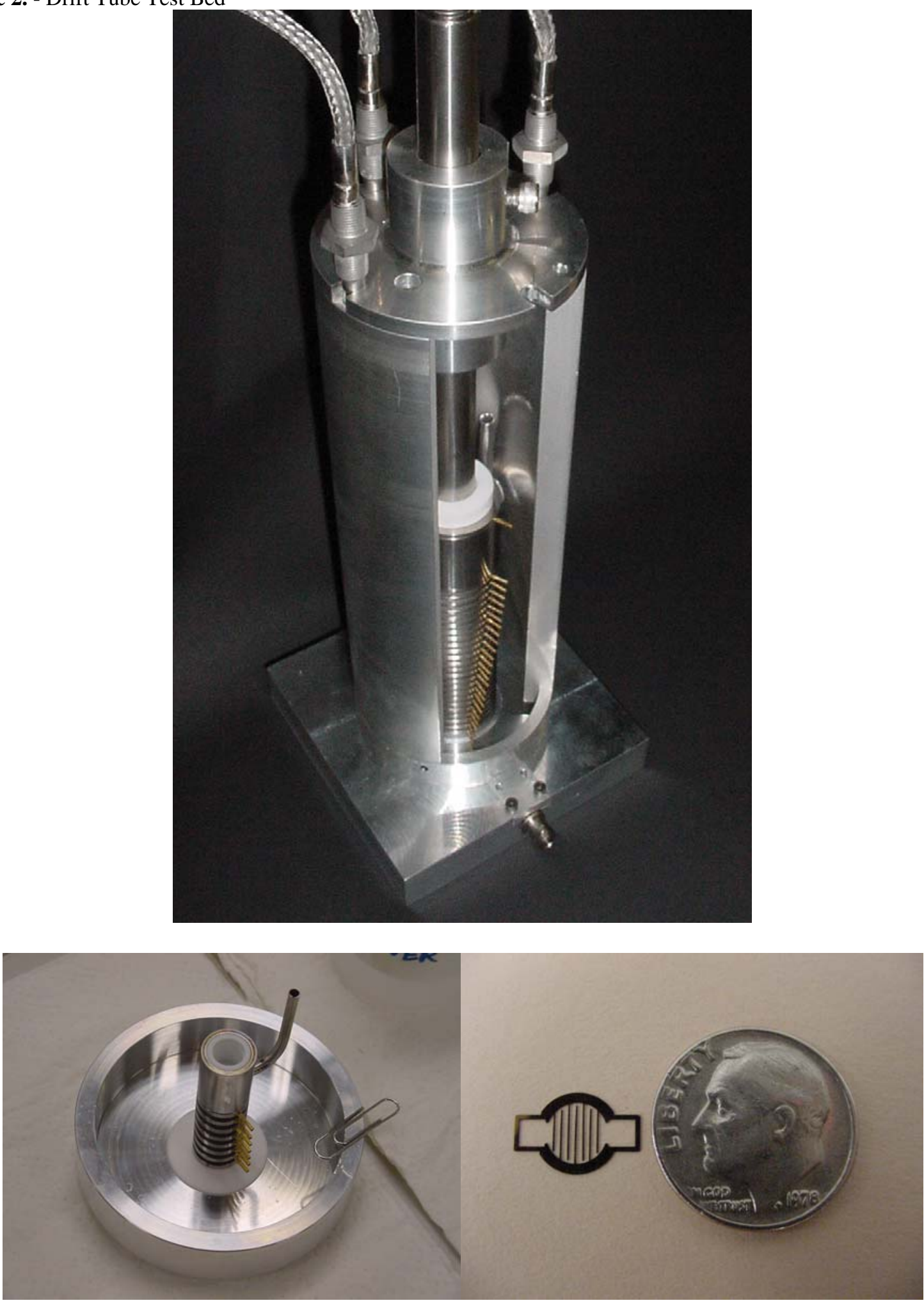

Top: Test bed with heating rods inserted: Shown here with large diameter, 30-drift ring tube.Lower Left: 10-drift ring, small diameter drift tube. Lower Right: Small diameter ion gate. (Note: Test Bed is displayed without access door, electrical connections and outer insulation) 


\section{Section III: Size Evaluation of Drift Tubes for Fourier Transform Ion Mobility Spectrometer}

\section{Executive Summary}

\section{Findings:}

- Ion transmission decreased with decreasing internal diameter. The smallest tube did not transmit ions.

- There was a minimal effect on reactant ion peak intensity and resolving power with the primary fluctuations being attributed to small changes in temperature and pressure that result from altering the drift gas flow rate.

- The resolving powers of the large and medium size tubes were not significantly different although the largest tube produced a slightly higher resolving power.

- While the observed resolving power was low compared with the theoretical maximum, these tubes did provide resolving powers similar to or better to those obtained in other small IMS tubes.

\section{Conclusions:}

- The width of the drift rings was too large, which produced a discontinuous drift field. This discontinuous drift field results in low ion transmission and reduced resolution power relative to that expected for tubes of this internal size.

- Temperature fluctuations as a result of varying drift gas flow rates can cause a drift in the measured mobility constants.

\section{Recommendations:}

- Of the three tubes tested the middle size provides the best compromise among resolving power, sensitivity, and size. This tube should be used for a handheld instrument.

- In future designs, the rings of the tube should be thinner to provide a smooth electric field. A smooth electric field will aid in increasing both resolving power and sensitivity.

- Given the practical limitations of a handheld instrument, the operating temperature and voltage should be $150 \mathrm{C}$ and $2000 \mathrm{~V}$, respectively.

\section{Project Objectives:}

The original objective of this project was to evaluate three specific drift tubes designed by Dr. Tarver at the Sandia National Laboratory for the application of these tubes in a handheld Fourier 
Transform Ion Mobility Spectrometer. In addition to experimental evaluation Dr. Herbert Hill of Washington State University was available to provide consulting for the overall project.

Initial Experiments consisted of assembling the tubes which were supplied by Sandia into a laboratory built oven for temperature control and testing. For testing purposes, Dr. Hill used electronics that he had developed at WSU. New electronics were not developed for this project. The electronics were borrowed from another IMS instrument in the laboratory for testing of the Sandia tubes. Several of the components did not work and needed to be fixed by the WSU electronics shop before the project could be started. Gates and gate drivers for these tubes were constructed and were attempted to be compared with those supplied by Sandia. However, the Sandia gates were of a design not readily compatible with the experimental setup. Specifically, the gates provided by Sandia were constructed using a lithography process which produced exposed gate wires that were structurally unsound. The ionization sources used for this project were supplied by WSU.

Once the electronics and testing assembly were constructed and operating properly, each tube was evaluated and compared with each other and with the IMS tubes currently operating in our laboratory.

Initial tube evaluation included stability of back ground current, noise under several gate and tube on/off positions, optimization of resolution and sensitivity for voltage, drift gas flow rate, gate timing. In addition to resolution and sensitivity for the standard, comparison of mobility constants were made to insure that the tube is operating correctly. Both short term and long term reproducibility for mobility constants, sensitivity, noise, back ground signal, and resolution were be monitored over a one month period.

A mid-project report was complied and a recommendation with respect to which of the three tubes should be used for the hand held device. Advantages and disadvantages of each of the tubes will be discussed.

During the second half of the project, various sample inlet designs will be considered and alternate drift gases will be evaluated for use in these tubes. The alternate gases will be evaluated as was the air drift gas above.

The final report did not include experimental findings of the alternate drift gas studies and several designs for sample introduction systems due to the expiration of funding.

\section{Evaluation of Objectives:}

The major objectives have been achieved during the course of this investigation. Electronics were modified and a test bed was constructed for the testing of the Sandia tubes. The experimental design and test bed for these experiments are given in the following section. The time line for the project did slip a few months for several reasons: First, the funding was not available on July 1 and spending on the project did not begin until October of 2003. A kickoff meeting was held with Dr. Tarver here in Pullman in September. The graduate student who was 
in charge of the project went on maternity leave in November due to the early arrival of her child. Brain Clowers took over the project at that time, finished the design and construction of the test bed and electronics. He began experiments in January. At the request of Dr. Tarver, this final report is being submitted in April, 2004 rather than June, 2004 as scheduled in the initial time line.

\section{Experimental Setup for Testing IMS Tubes:}

To accommodate the varying diameters of the drift rings supplied by Sandia National Laboratories a test bed shown in Figure 1 was designed and constructed. Briefly, a ${ }^{63} \mathrm{Ni}$ source was placed $\sim 1 \mathrm{~cm}$ from the ion gate and the only source explored for ionization. The ion gate employed was of a Bradbury-Nielsen configuration and was constructed here at WSU. The ion gate was interfaced to the smaller diameter drift rings using a Macor spacer as shown in Figure 1. A stainless steel Faraday plate was used for detection of ions after they traversed the $5.9 \mathrm{~cm}$ drift tube. All three drift tube designs (0.578”, 0.300”, and 0.137” I.D.) provided by Sandia were evaluated and the data shown below. Currently, the ion mobility instrumentation at Washington State University uses a design similar to the tubes provided by Sandia, however, the inner diameter is significantly larger: 1.88". A drift tube using the WSU design was also used to compare the performance between the different systems.

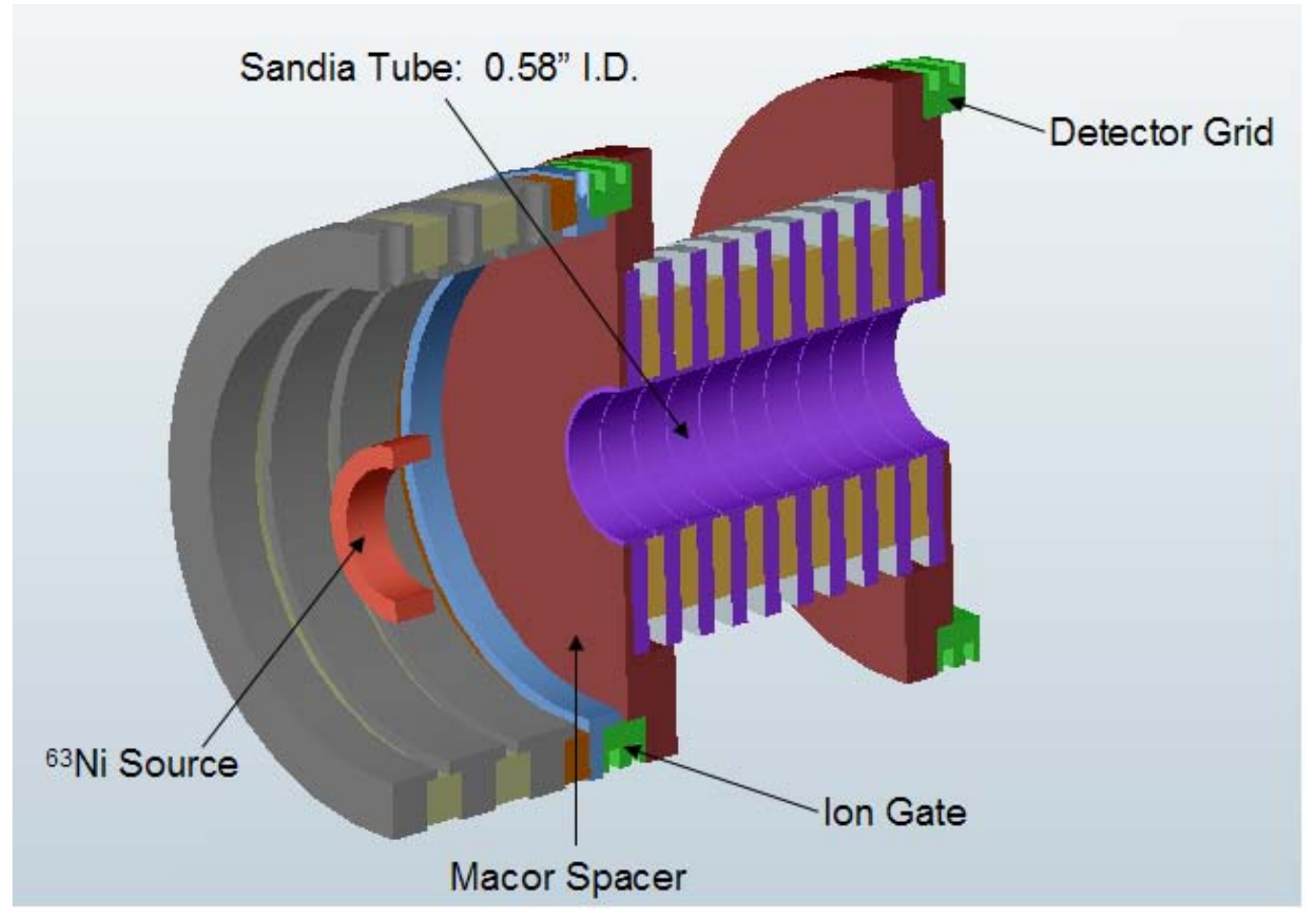

Figure 1. - Source, Ion Gate and Drift Tube Schematic

While maximum ion mobility resolving powers are achieved at lower temperature, minimal gate pulse width and optimum drift potential, operation under these conditions often requires a 
sacrifice in sensitivity. For the systems studied, peak intensities and resolving powers were examined as a function of drift potential, temperature, drift gas flow rate, and ion gate pulse width. To examine the role of temperature on the resolving power of each system, the temperature was systematically lowered starting at $150^{\circ} \mathrm{C}$ down to $70^{\circ} \mathrm{C}$ in $20^{\circ} \mathrm{C}$ increments. At each individual temperature value the effect of ion gate voltage on the resolving power and drift time of reactant ions produced by the ${ }^{63} \mathrm{Ni}$ was explored $(2000 \mathrm{~V}-500 \mathrm{~V})$. Additionally, the effect of gate pulse width $(0.1-0.4 \mathrm{~ms}$ pulses $)$ on intensity and resolution was examined at select temperatures and drift potentials. Each spectrum is the average of 1000 individual spectra. The final stage of this study was to examine the effect, if any, on the drift time and resolving power of the reactant ion peaks by varying the counter-current drift gas flow rate.

\section{Results and Discussion:}

Figure 2 illustrates the change in both intensity and drift time of the reactant ion peak as a function of the drift potential applied across the large diameter ion mobility system. All peaks obtained at voltages above $1250 \mathrm{~V}$ are clearly distinguished in the spectrum; however, those acquired under lower field conditions are limited in their intensity and are difficult to distinguish from the background noise.

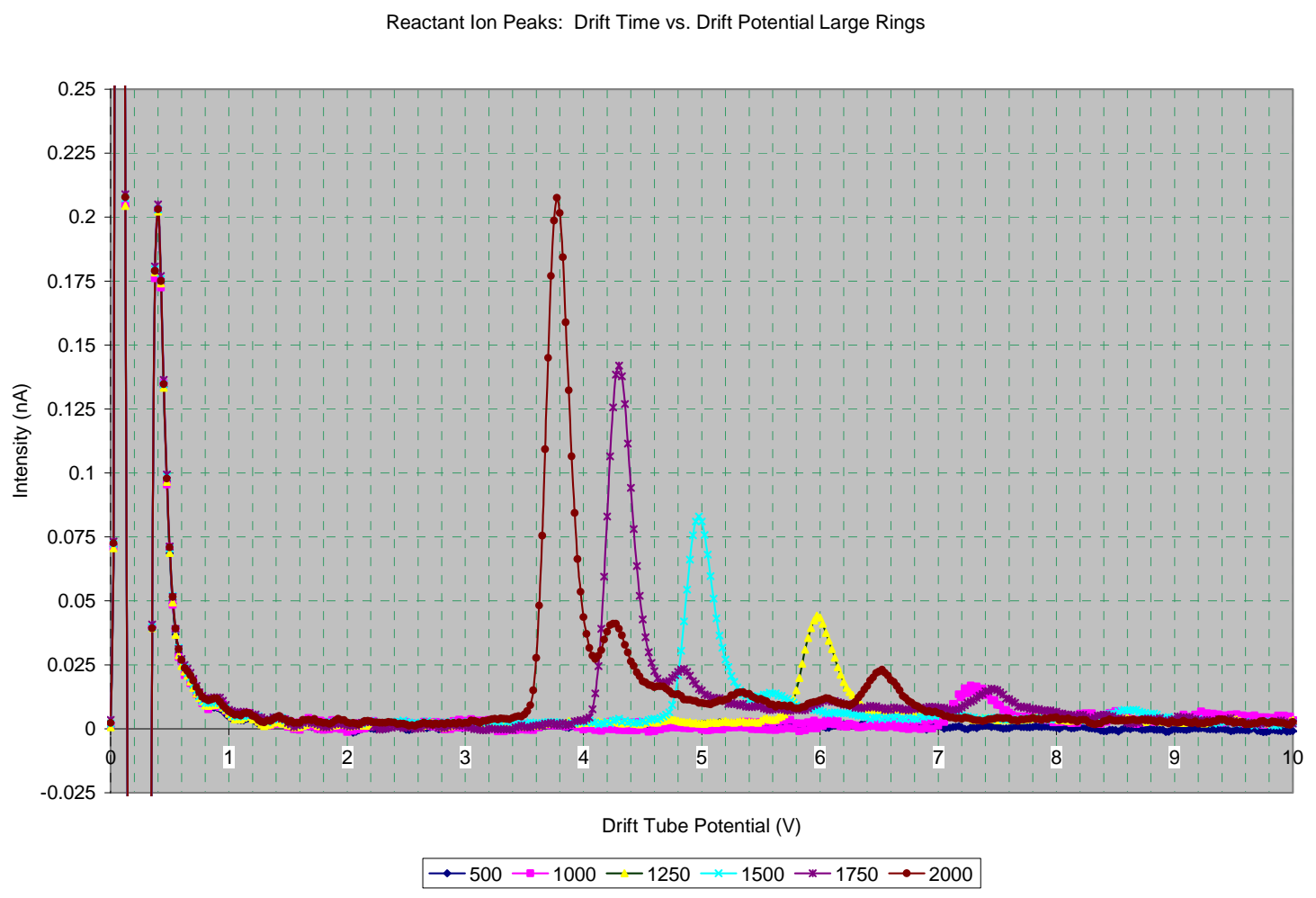

Figure 2. - Reactant Ion Drift Time vs. Drift Voltage

Figure 3 illustrates the effect of drift gas flow rate on peak intensity and drift time when the large ring systems were held at $150^{\circ} \mathrm{C}$ and operated at a drift potential of $2000 \mathrm{~V}$. A slight variation in 
drift time is observed over the range of drift gas flow rates (260-1625 $\mathrm{mL} / \mathrm{min})$, however, this is attributed to a change in temperature that occurs with large variations in drift time. Overall, for the same temperature and drift potential the mean drift time of the reactant ion peak determined with the large tube system was $3.82 \pm 0.06_{5} \mathrm{~ms}$, and for the medium ring system $3.92 \pm 0.05_{5} \mathrm{~ms}$. It should be noted that while these drift times between systems appear significantly different; these numbers have not been normalized for the variations in ambient pressure on the days each data set was acquired. Another attribute of note is the growth of a second reactant peak when flow rate is increased. At higher drift gas flows the small peak shoulder located to the right of the main reactant ion peak grows to a well developed peak. This peak growth is believed to be a result of the small variations in temperature and pressure that are accompanied by a change in flow rate their influence on the clustering behavior of specific ion populations.

Drift Time Variation as a Function of Drift Gas Flow Rate: Large Rings, 2000V, 0.2ms PW $150 \mathrm{C}$

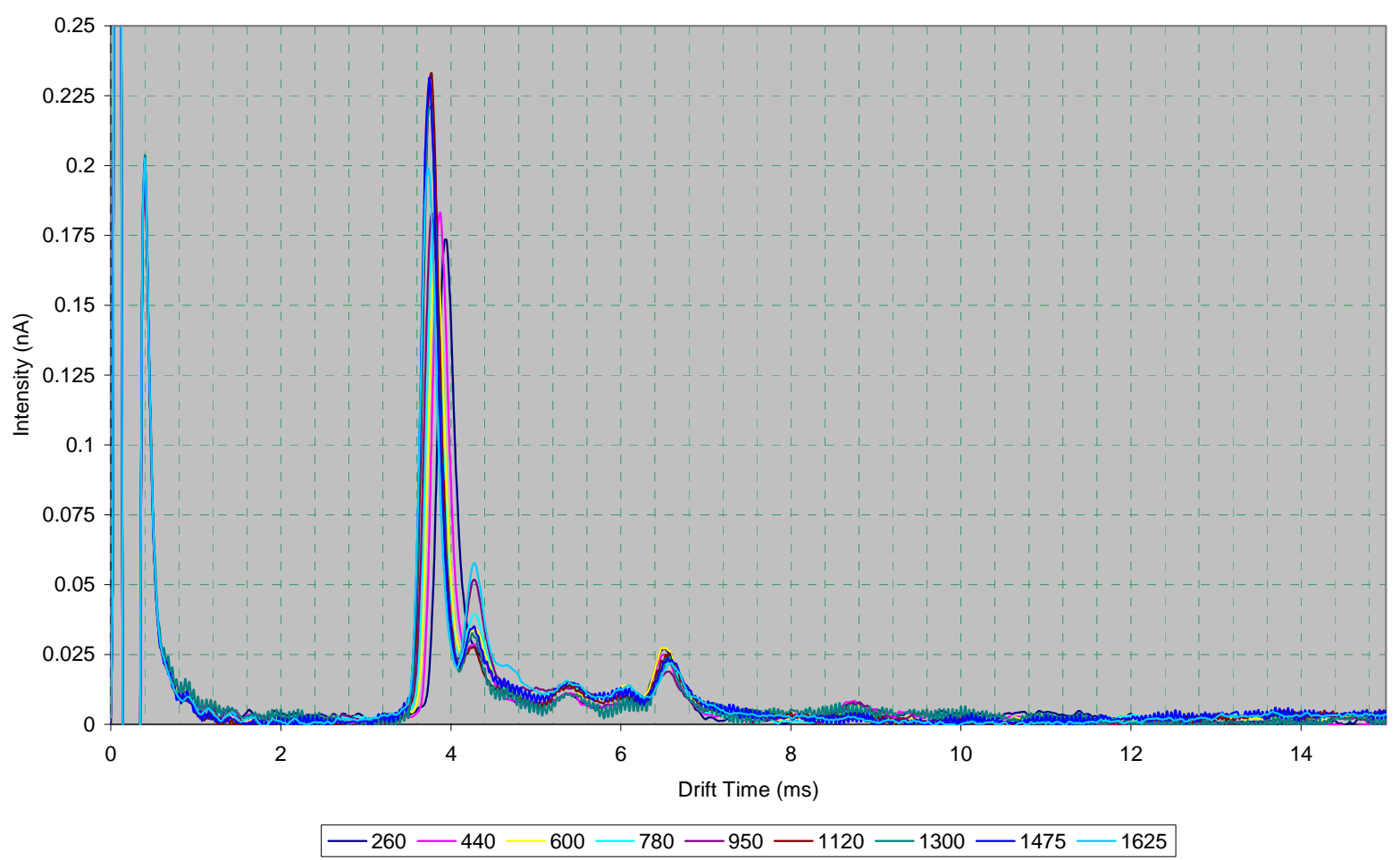

Figure 3. - Drift Time vs. Flow Rate (large rings)

Figures 4 and 5 summarize the effects of drift gas flow rate on reactant ion peak resolving power and intensity. Figure 4 corresponds to the data acquired for the large ring system, whereas, Figure 5 are the results for the same study performed on the medium ring system. For both the large and medium ring size system, the resolving powers did not fluctuate radically. This indicates a minimal, if any, effect of flow rate on the resolving power of the system. The resolving power for the primary reactant ion peak for the large and medium rings systems were 
$16.88 \pm 0.43$ and $14.32 \pm 0.38$, respectively. For the large rings system a small increase in peak intensity at higher flow rates was observed, however, such high drift gas flow values are not practical for field use and were primarily acquired to provide a more complete picture. Taken as a whole, this drift gas flow study indicates a minimal effect on reactant ion peak intensity and resolving power with the primary fluctuations being attributed to small changes in temperature and pressure that result from altering the drift gas flow rate.

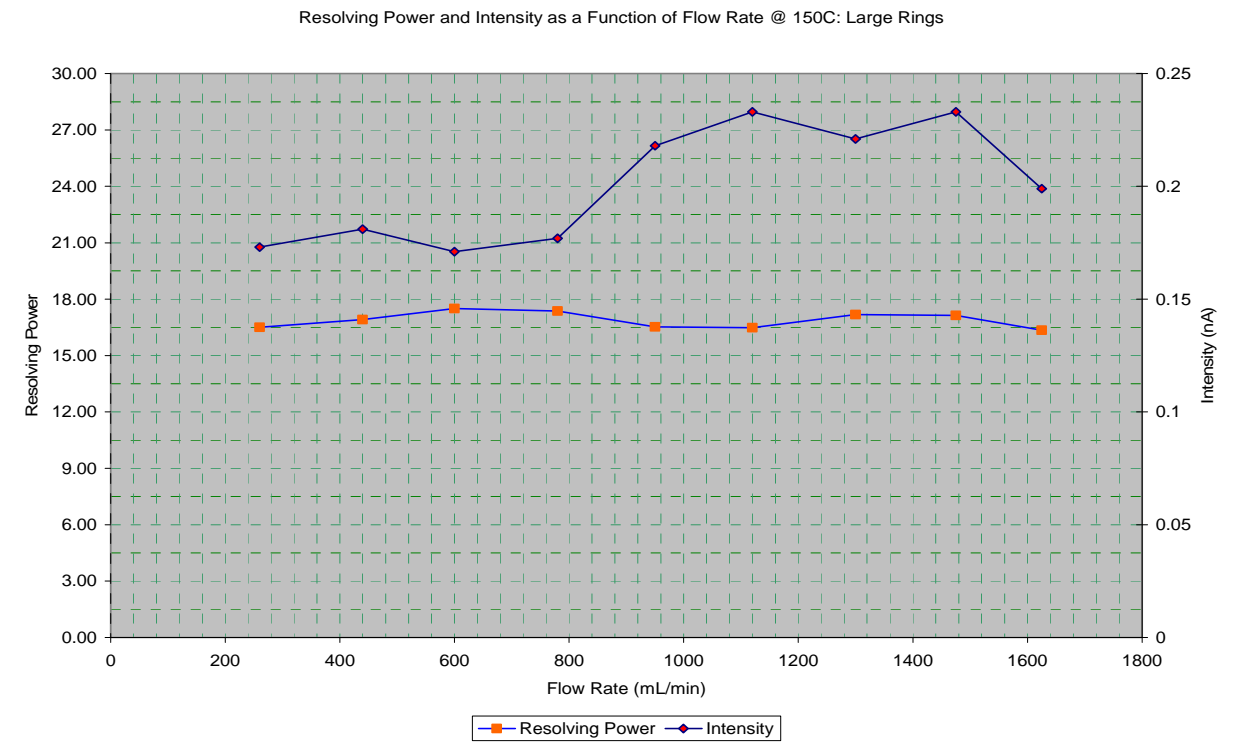

Figure 4. - Resolution vs. Flow Rate (large rings)

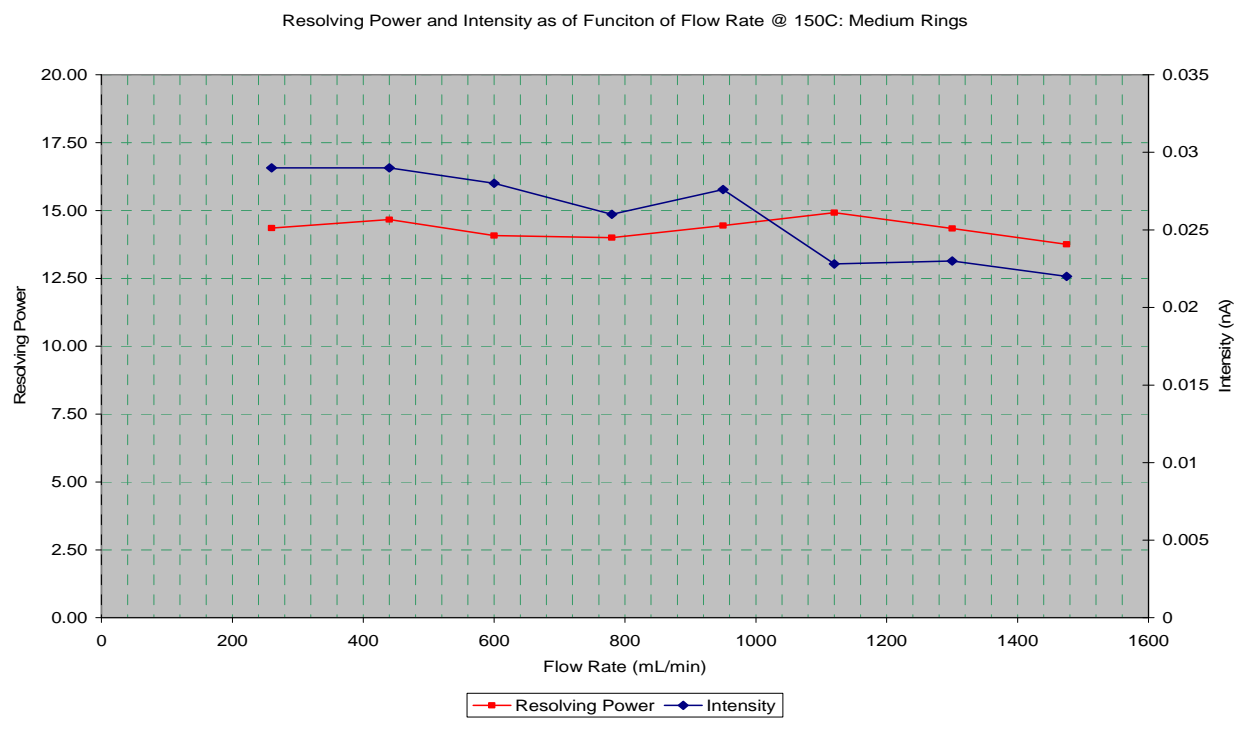

Figure 5. - Resolution vs. Flow Rate (medium rings) 
Figures 6, 7, and 8 compare the resolving power as a function of drift potential for both large, medium, and WSU rings. The various data sets were acquired under specific voltage and temperature conditions while holding the flow rate constant throughout the measurements. For the Sandia tubes the highest resolving power ( 23) was obtained for the large drift rings when operating at $70^{\circ} \mathrm{C}$ - the lowest temperature at which data was obtained. The larger diameter of the WSU rings, on the other hand, produced somewhat higher resolving powers ( 29) also at $70^{\circ} \mathrm{C}$. Comparing the two Sandia tube sets, the large rings produced a slightly higher resolving power than the medium rings. The mean resolving powers for the WSU, large and medium drift rings across all the experimental variables were $23.34 \pm 4.01,18.31 \pm 3.07$ and $15.48 \pm 2.16$ respectively.

Resolving Power as a Function of Drift Voltage: Large Rings

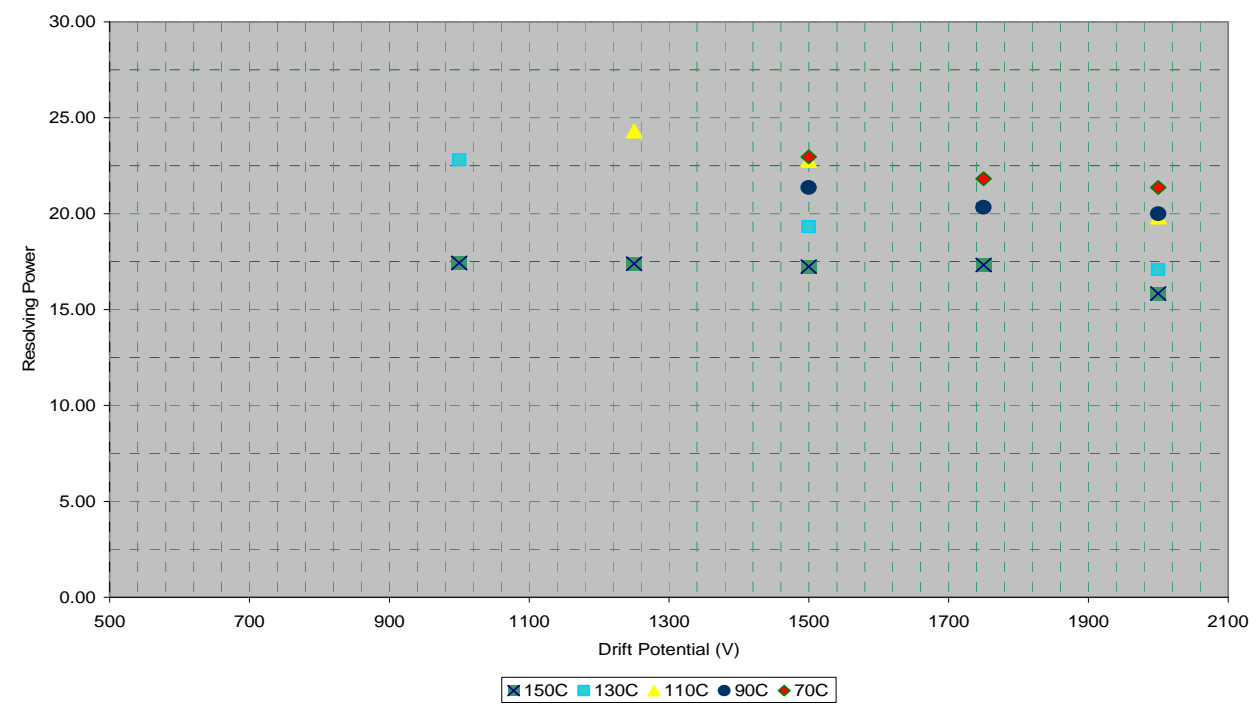

Figure 6. - Resolution vs. Drift Voltage (large rings)

Resolving Power as a Function of Drift Potential: Medium Rings

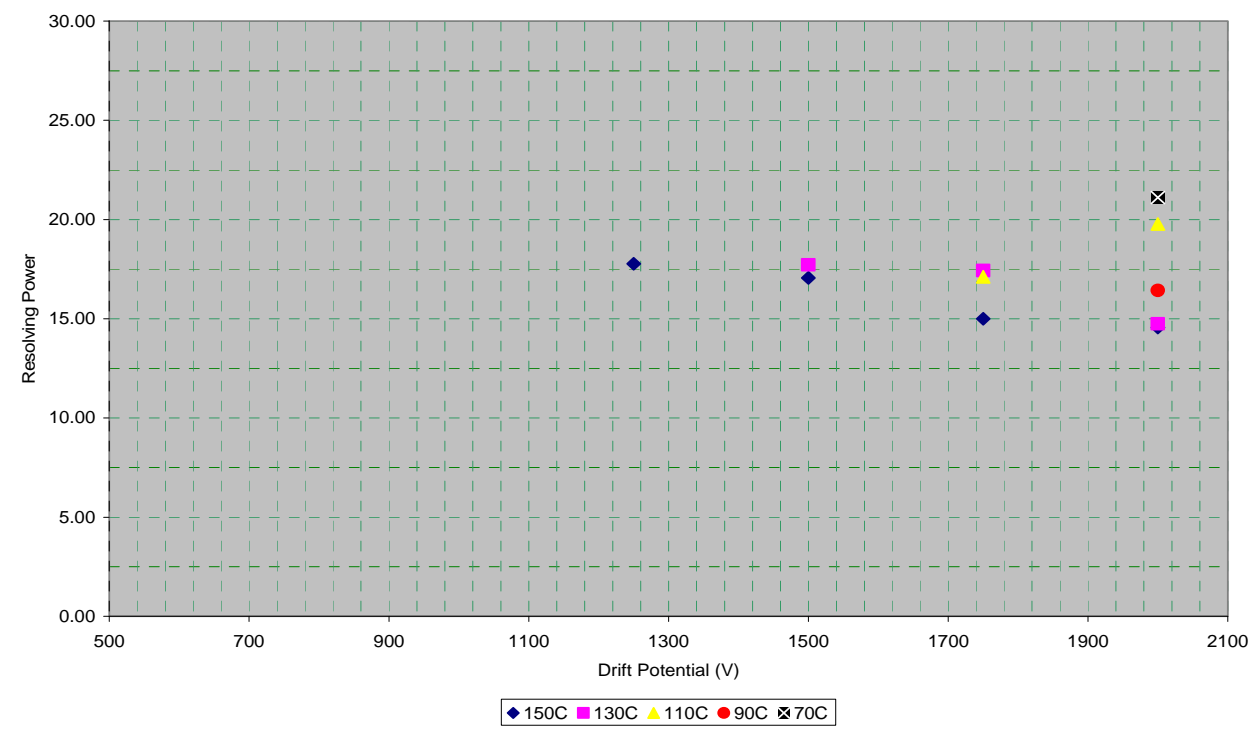

Figure 7. - Resolution vs. Drift Voltage (medium rings) 


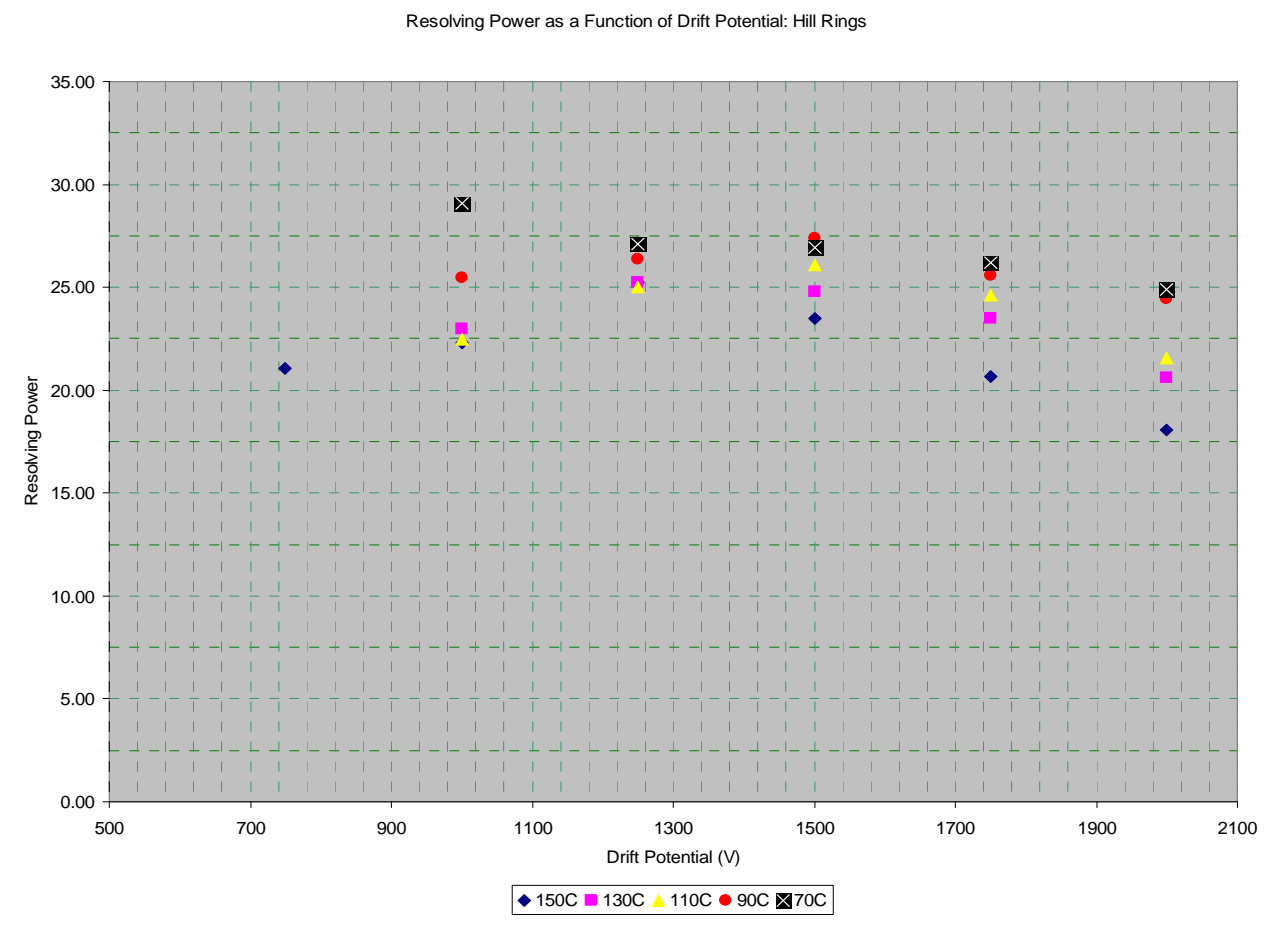

Figure 8. - Resolution vs. Drift Voltage (WSU rings)

Figure 9 compares the experimentally determined resolving powers of the reactant ion peaks obtained for the WSU, large, and medium rings to the theoretical maximum. The reactant ion peaks were measured under the following conditions: $200^{\circ} \mathrm{C}, 2000 \mathrm{~V}$ drift voltage, and 780 $\mathrm{mL} / \mathrm{min} \mathrm{N}_{2}$. When examining the Sandia tube sets repeated attempts were made to account for the deviation of the experimental results from the theoretical maximum resolving power; however, a definitive cause has yet to be identified. Given the high concentration of ions produced by the ${ }^{63} \mathrm{Ni}$ source and the small diameter of the drift tubes, space charge effects and field heterogeneity may be the cause. To expand upon this conclusion, compared to the width of each stainless steel drift ring the insulating space is small. The result is a disjointed electric field gradient. Rather than creating a smooth gradient for the ions to migrate, this configuration establishes a gradient resembling a staircase. This "staircase" gradient or field heterogeneity disrupts the flow of ions and contributes significantly to deviations from the theoretical resolving power. When the drift potential is operated above $\sim 1400 \mathrm{~V}$ the WSU drift rings agree with the resolving power values predicted by theory. A marked cut-off of ion transmission for all of the tubes used in the study occurred at drift region voltages below $\sim 1000 \mathrm{~V}$. 


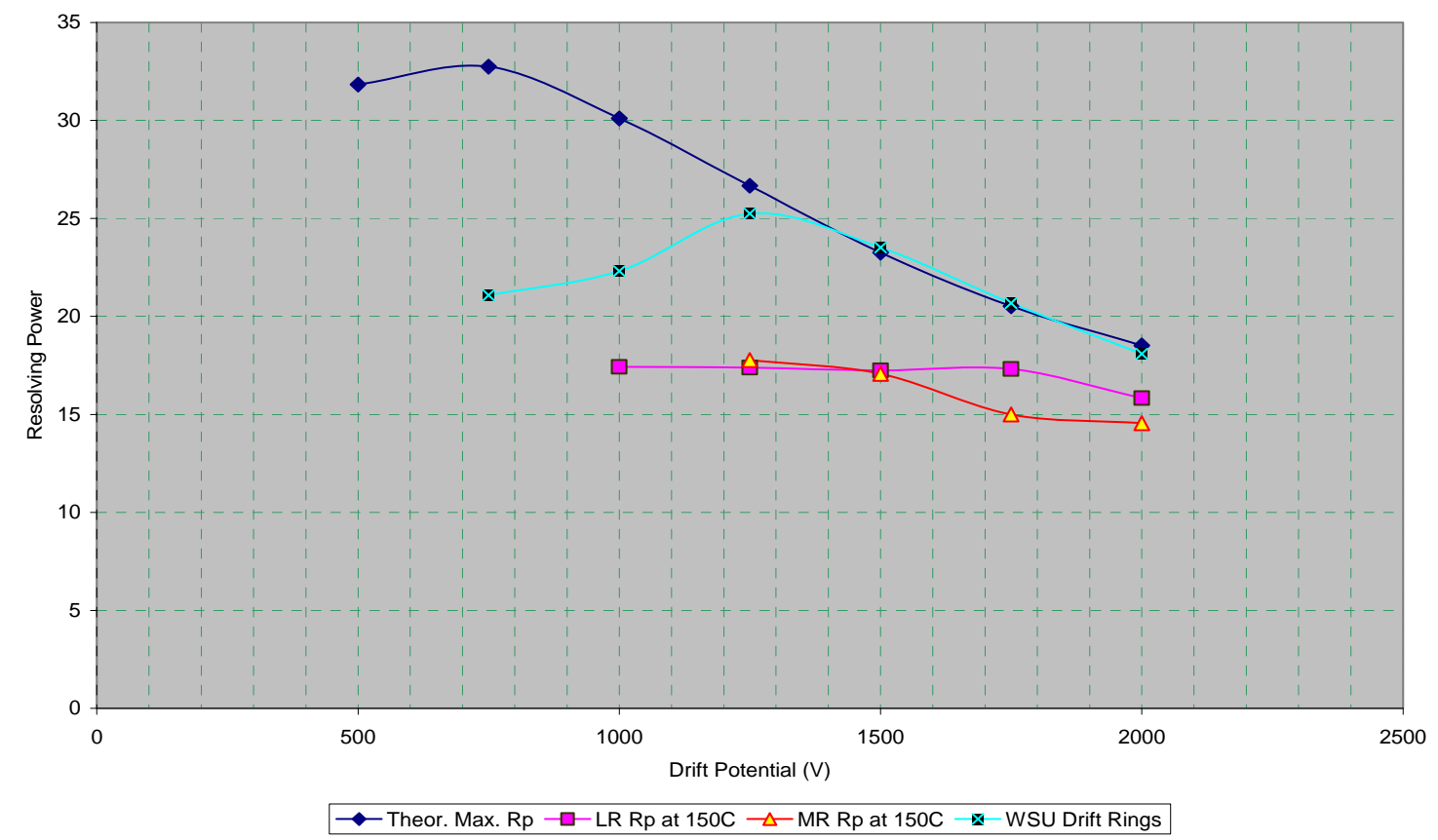

Figure 9. - Theoretical vs. Experimental Resolution

The following set of graphs, Figures 10 and 11, track the reactant ion peak intensity as a function of both drift potential and temperature. As discussed earlier, operating at lower temperatures and optimum drift tube potentials optimizes resolving power but diminishes instrumental sensitivity. These experiments showed that the maximum peak intensity was achieved at $150^{\circ} \mathrm{C}$ and a drift potential of $2000 \mathrm{~V}$ (flow rate did not significantly alter peak intensity). It should be noted that

Peak Intensity as a Function of Drift Voltage: Large Rings

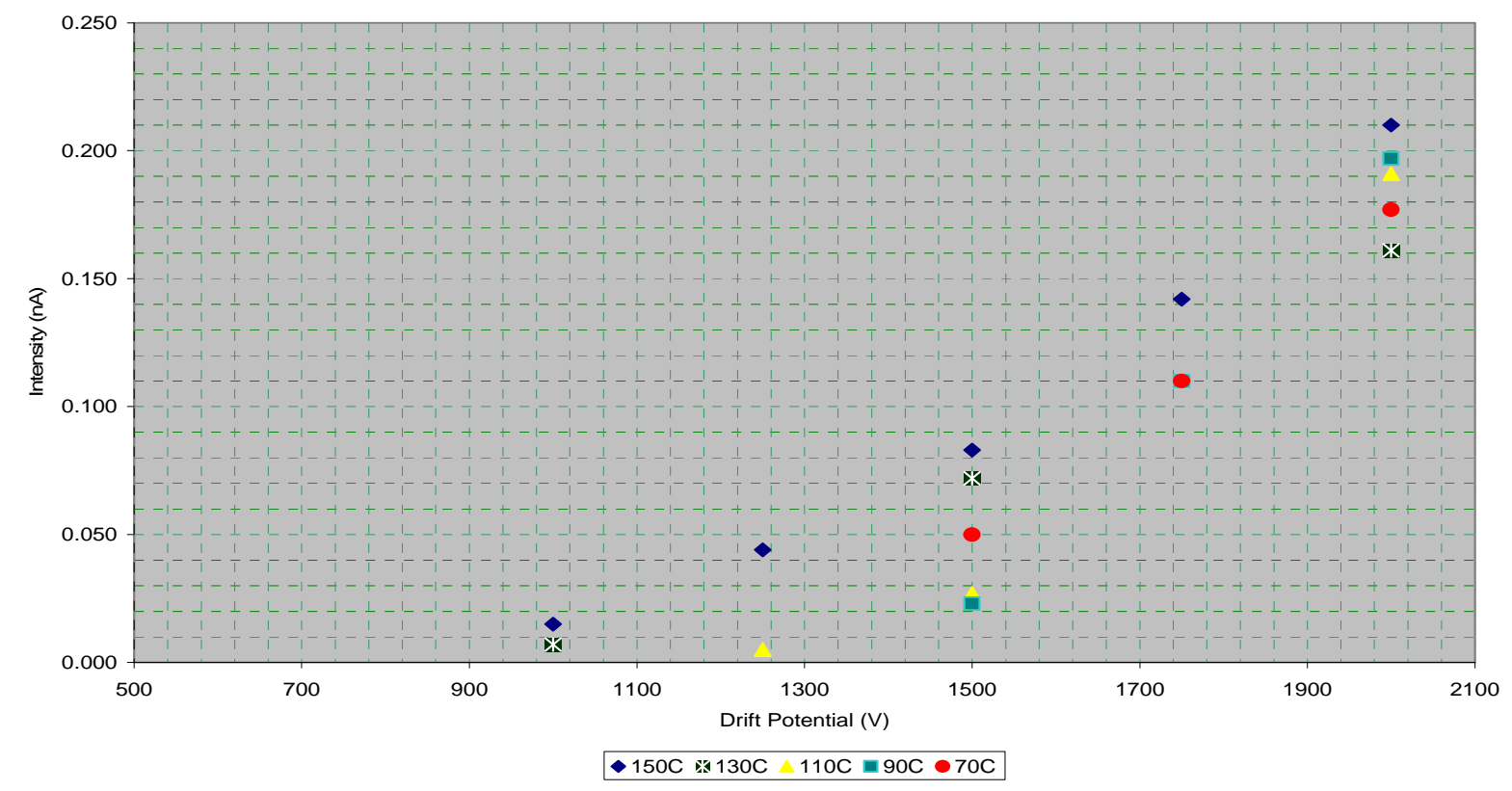

Figure 10. - Peak Intensity vs. Drift Voltage (large rings) 
Figures 10 and 11 are not normalized for the drift tube cross sectional different between the large and medium tubes. The peak intensities obtained with the medium drift tube system were normalized by multiplying by the ratio of cross sectional area between the large and medium tubes. The resulting values are plotted in Figure 12. Despite this multiplication factor the normalized peak intensity values fall short of those obtained for the larger system under similar conditions. This further demonstrates the negative implications of tube diameter minimization. It is suggested that a proportional minimization procedure be explored for smaller drift tubesthe ratio by which the diameter is reduced must also be applied to ring spacing and width.

Peak Intensity as a Function of Drift Potential: Medium Rings

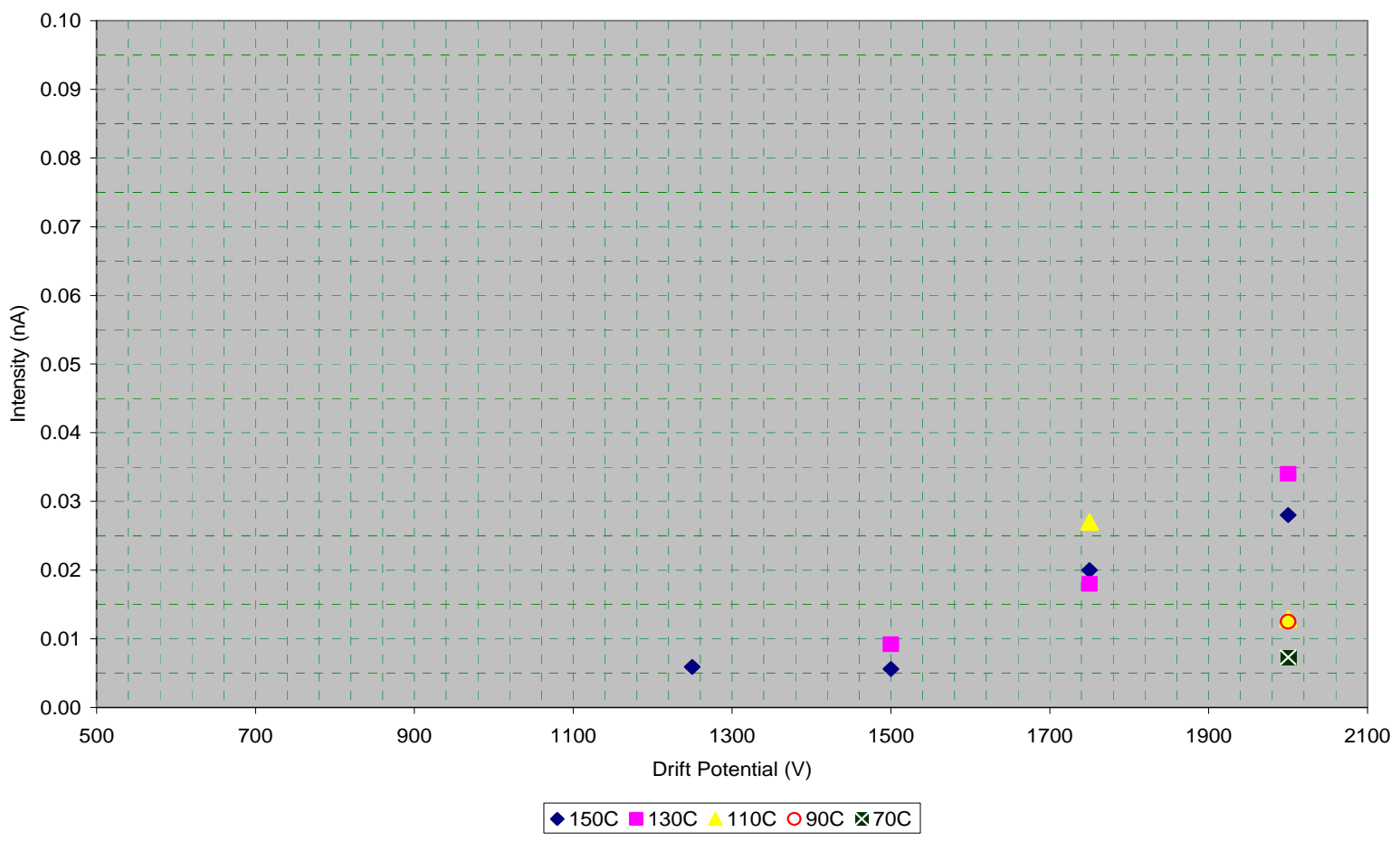

Figure 11. - Peak Intensity vs. Drift Voltage (medium rings) 
Peak Intensity as a Function of Drift Voltage: Hill Rings

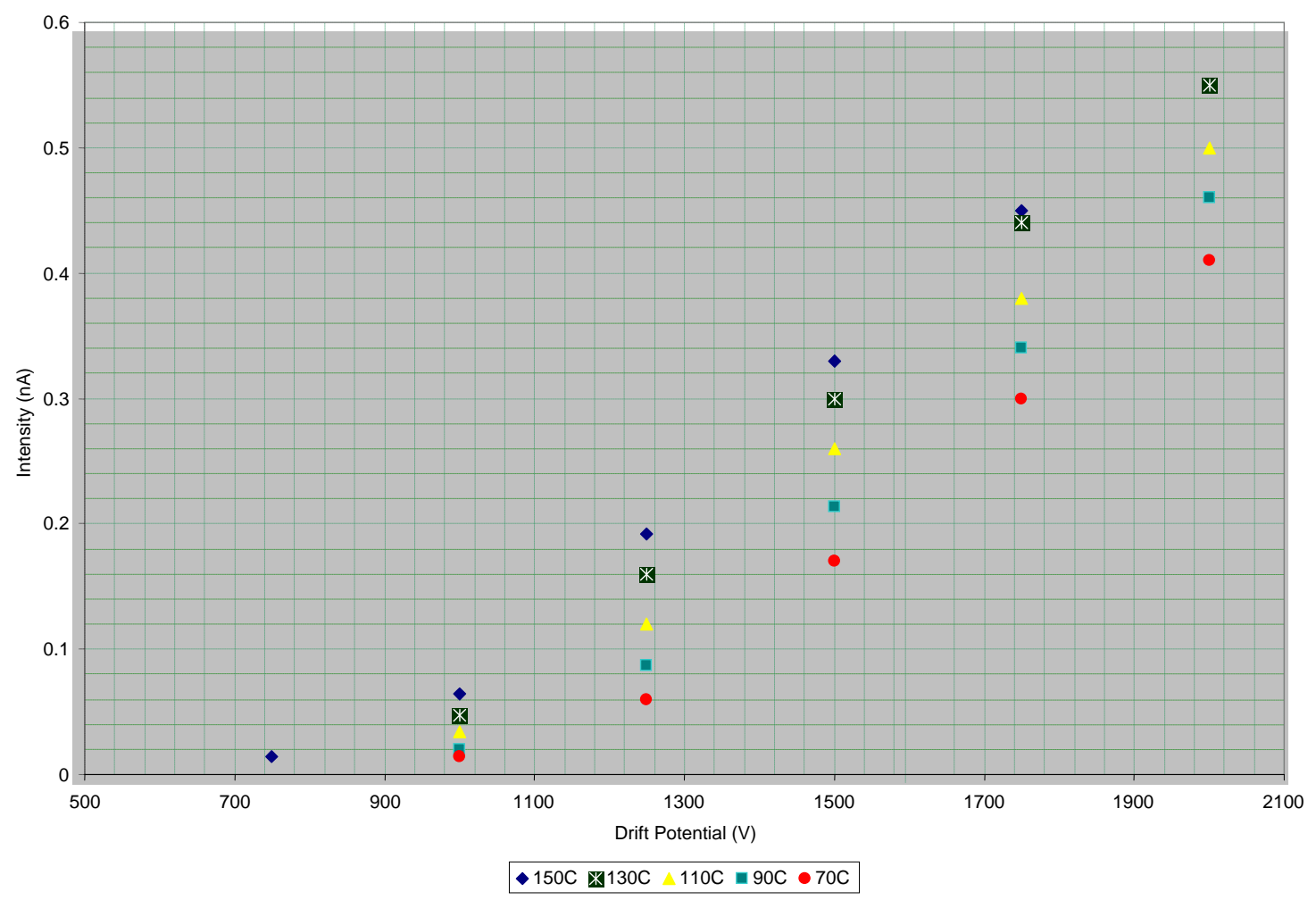

Figure 12. - Peak Intensity vs. Drift Voltage (WSU rings)

Peak Intensity as a Function of Drift Potential @ 150C:

Comparison of Hill Rings, Large Rings, Medium Rings, and Medium Ring Intensity Adjusted for Collector Area

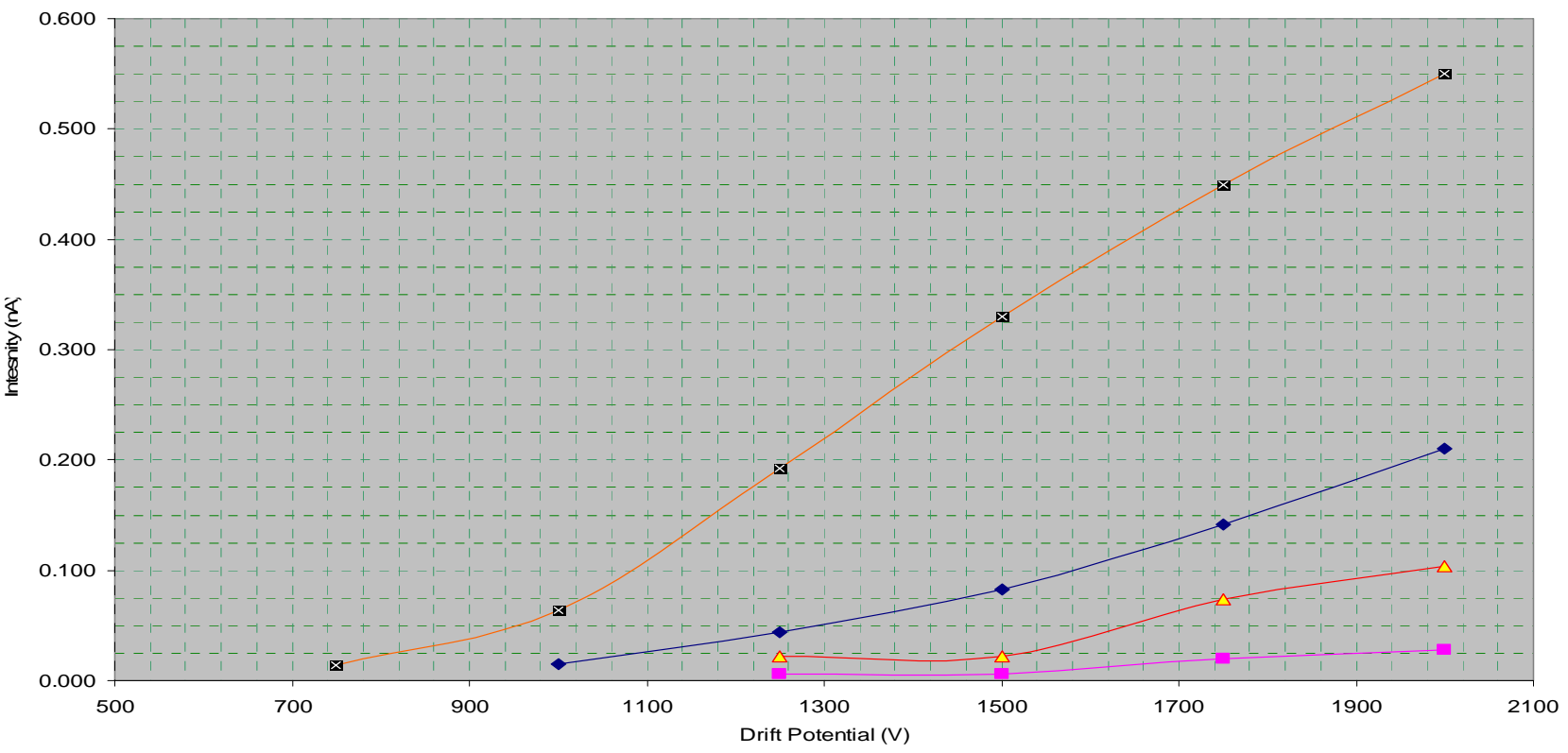

LR $150 C \rightarrow-M R$ 150C $\triangle M^{\star} R^{\star} 150 C-$ Hill Drift Rings

Figure 13. - Peak Intensity vs. Drift Voltage $\left(150^{\circ} \mathrm{C}\right)$ 
Large Rings: Drift Potential vs. Resolving Power and Peak Intensity @ 150C

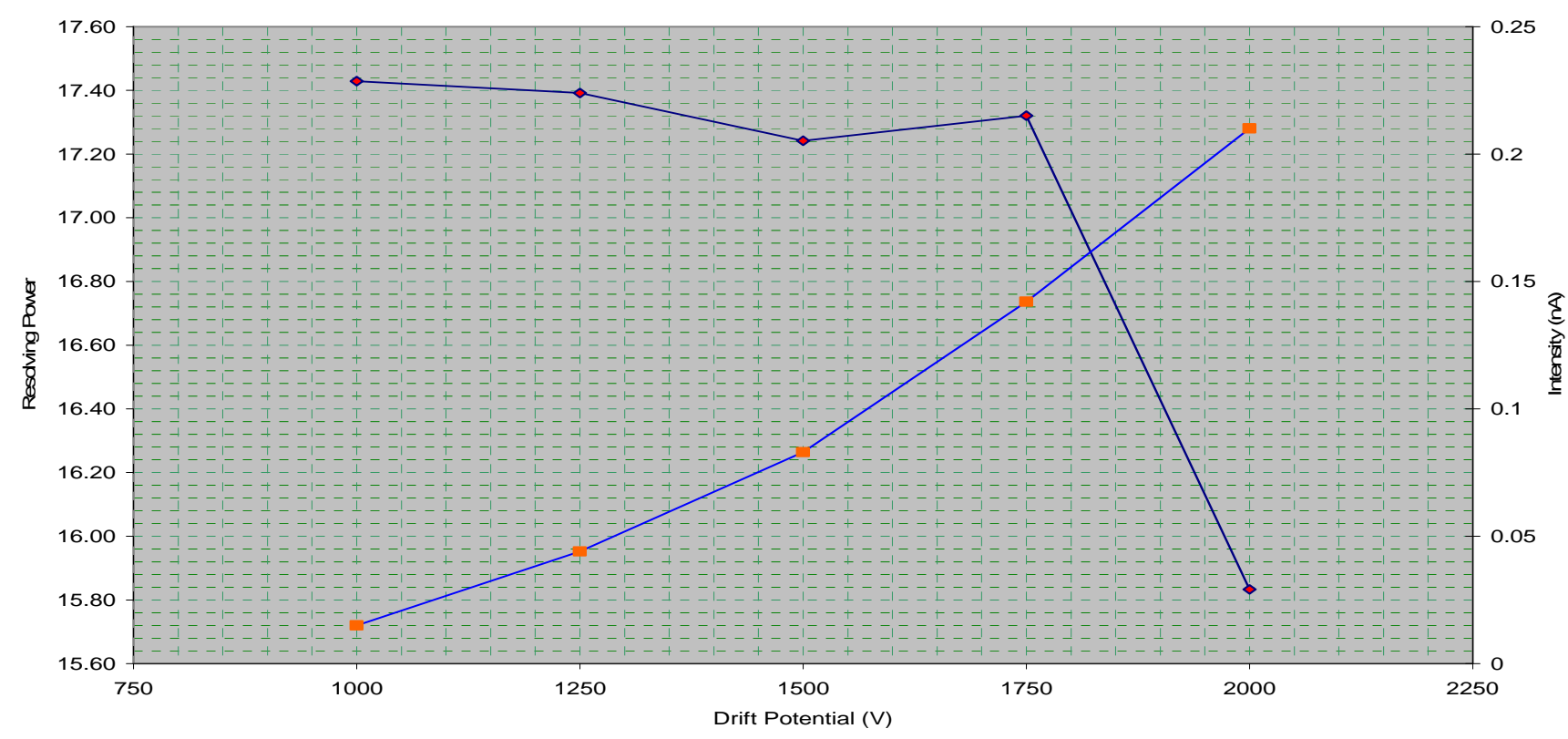

Figure 14. - Drift Voltage vs Resolution $\left(150^{\circ} \mathrm{C}\right)$

\section{Summary and Conclusions:}

The performance of three ion mobility tubes supplied by Sandia National Laboratories was evaluated. The largest (0.578”) and medium (0.300”) diameter tubes were the only systems found to provide the necessary ion transmission necessary for data acquisition—data regarding peak shape and behavior of ions in the smallest ion mobility tube was not available. Resolving powers of $\sim 18$ were routinely achieved for both medium and large diameter IMS systems', however, the peak intensity obtained with the larger diameter system was significantly greater than that provided by the medium diameter system. As flow rate played a minor role in the performance of the system, this is a potential factor that may be altered to minimize resource usage in the field. Overall, the larger diameter IMS system provided the best balance between peak intensity and resolving power. In order to miniaturize the IMS systems even further it is recommended that ratio by which the internal diameter is reduced must also be applied to the spacing and width of the individual drift rings and insulators. This system-wide reduction will help scale down the electric field geometry to accommodate more efficient ion transport. 


\section{Distribution}

\section{UNCLASSIFIED Unlimited Release Documents:}

$1 \quad$ MS 9001

1

$1 \quad$ MS 9403

$1 \quad$ MS 9403

$1 \quad$ MS 9403

$1 \quad$ MS 9101

$1 \quad$ MS 9102

$1 \quad$ MS 1425

$3 \quad$ MS 9018

$1 \quad$ MS 0899

$1 \quad$ MS 9021
John, M.E., - 8000

Attn: Stulen, R. H. - 8100, MS 9004

Henson, D.R. - 8200, MS9007

McLean, W.J. - 8300, MS 9054

Smith, P.N. - 8500, MS 9002

Hruby, J. - 8700, MS 9405

Washington, K.E. - 8900, MS 9003

Wilson, Ken - Department 08770

Attn: Spence, Paul, Department 08774

Cadden, Chuck, Department 08772

Tarver, Ed - Department 08773

Wang, Jim - Department 08773

Phan, Anh - Department 08773

Jennings, Richard - Department 08232

Stamps, Jamie - Department 08235

Pfeifer, Kent - Department 01744

Central Technical Files, 8945-1

Technical Library, 9616

Classification Office, 8511 for Technical Library, MS 0899, 9616

DOE/OSTI via URL 